\title{
Dynamic Molecular Interactions Between Polyurethane and ZIF-8 in a Polymer-MOF Nanocomposite: Microstructural, Thermo-Mechanical and Viscoelastic Effects
}

\author{
E. M. Mahdi and Jin-Chong Tan* \\ Department of Engineering Science, Parks Road, OX1 3PJ, \\ University of Oxford, United Kingdom. \\ *jin-chong.tan@eng.ox.ac.uk
}

\begin{abstract}
Polymer-based nanocomposites incorporating porous hybrid materials is fast becoming a staple in addressing environmental and sustainability challenges. Herein we investigate the intricate dynamics underpinning the molecular interactions between the porous nanoparticles of a zeolitic metal-organic framework (ZIF-8) and a hyperelastic polymer matrix (polyurethane, PU). We fabricated novel PU/ZIF-8 mixed-matrix nanocomposite membranes ( 100 $\mu \mathrm{m}$ thickness) with up to $40 \mathrm{wt}$.\% ZIF-8 nanoparticles, and characterised their quasistatic and dynamic thermo-mechanical properties via dynamic mechanical measurements, nanoindentation, thermogravimetric and tensile experiments. ZIF-8 nanoparticles significantly modify the thermo-mechanical properties of polyurethane matrix, improving overall stiffness, fracture energy, and yield strength at the cost of ductility (beyond 30 wt.\%). Thermal stability was enhanced, likewise dynamic responses and phase changes were stabilised with increasing nanoparticle loading. Secondary molecular interactions between ZIF-8 nanoparticles and polyurethane chains are responsible for the salient thermomechanical and dynamic behaviour observed. Our findings may guide developments of resilient rubbery-MOF nanocomposite systems for practical applications.
\end{abstract}

Keywords: polyurethane nanocomposite; zeolitic imidazolate framework; viscoelasticity; thermo-mechanical behaviour 


\section{Introduction}

Recent advances in polymer science and chemistry alongside the advent of hybrid inorganic-organic materials offers a unique and innovative opportunity/solution for current problems plaguing the globe, such as environmental pollution [1], energy depletion [2], and the purification of vital resources, most importantly water [3]. Polymers, especially rubbery polymers (elastomers), are valued for their strechability [4], extended ductility (hyperelasticity) [5], and incompressibility [6]. They are made up of extremely long and loose molecular chains that are intertwined and loosely associated with one another via a collection of weak interactive bonds, with extended freedom of movement, mostly manifesting itself in the form of internal continuous motions of primary and secondary molecular polymeric chains [7]. This result in their distinctive and highly recognisable responses to external stimuli and malleable and highly extensible (ductile) nature [8]. They are also known for low to non-existent water absorption [9] and being electrically insulating (good dielectrics) [10]. This makes them perfect for applications such as food packaging [11] and protective clothing [12], tires for automobiles and aeroplanes [13], and heat shields [14]. This class of polymers could be synthesised via a variety of techniques, ranging from vulcanisation [15] to electrospinning [16].

Hybrid inorganic-organic materials are made up of multiple classes, with MetalOrganic Frameworks (MOF) [17] forming one of its major subclasses. MOFs are framework compounds made up of metallic ion centres coordinated by organic linkers, forming a repeating continuous 3D porous framework with large surface areas, increased accessible active sites, and low densities $[18,19]$. While they are regarded as structural relatives of inorganic compounds such as zeolites and silica, the chemical and structural variability of MOFs are significantly more diverse. In addition to coordination and strong covalent bonding that give rise to their 3D open-framework architectures, MOFs are further dominated by 
combined secondary chemical interactions such as hydrogen bonds (henceforth known as Hbonds), van der Waals forces (henceforth known as vdW forces), $\pi-\pi$ stacking [20]. MOFs can be formed using almost any combination of metallic (e.g. $\mathrm{Zn}^{2+}, \mathrm{Cu}^{2+}$ ) coordination environments connected together by organic-based linkers (e.g. imidazoles (Im), benzenedicarboxylates (BDC) and benzetricarboxylates (BTC)) via a multitude of physical or chemical techniques [21], making the possible structural permutations of MOFs almost limitless [22]. Many classes of MOFs are currently being studied, such as Zeolitic Imidazolate Framework (ZIFs) [23], HKUST-1 [24], and UiO-66 [25]. MOFs are considered as promising candidates for gas separation [26] and hydrogen/energy storage [27] applications. This is reflected in many reported works, amongst them Cacho-Bailo et al. [28], who discussed the synthesis of a continuous ZIF-93 membrane within the inner walls of P84 polymer channels that resulted in improved selectivity vis-à-vis $\mathrm{H}_{2}-\mathrm{CH}_{4}$ and $\mathrm{CO}_{2}-\mathrm{CH}_{4}$, and Xia et al. [29], who reviewed the applicability and recent work involving electrochemical storage and conversion involving a variety of MOF structures in relation to their respective advantageous intrinsic properties, such as large surface areas and tuneable pores. However, recent studies have explored new innovative applications of MOFs to afford drug delivery, multifunctional sensors, photoluminescence, and optoelectronics [30, 31].

The recent trend in exploring the viability and applicability of nanostructures is also evident in advances pertaining to MOFs $[32,33]$, where the synthesis, characterisation, and application of nano-MOFs are reported with increasing frequency, and has come to dominate MOF literature, due mostly to the established superiority of nano-MOFs over their micro, macro, and bulk counterparts $[34,35]$. Examples of this are ubiquitous in literature, among them Liu et al. [36], who discussed the enhanced gas storage capabilities of a Graphene Oxide (GO) membrane when nanosized Cu-MOFs fillers were introduced; this was attributed to the better dispersion of nano-Cu-MOFs as opposed to its bulk counterpart, which are prone 
to the formation of aggregates or agglomeration, forming distinct and separate phases within the matrix. Recently it was reported that two immiscible high-performance polymer blends (PBI and 6FDA) were compatibilised using ZIF-8 nanoparticles, resulting in a membrane microstructure featuring a uniform domain size [37]. This might be ascribed to lowering of interfacial energy due to localisation of ZIF-8 nanoparticles at the interface of the two immiscible polymers. The intrinsic advantage of nano-MOFs over its bulk or macro counterpart is also seen elsewhere, where Huxford et al. [38] reported the viability of nanoMOFs for drug delivery applications due to their smaller sizes, making it easier for them to enter and be circulated within the bloodstream and deliver drugs to cells as opposed to bulk MOFs, due to the fact that they will encounter a problem identified as irregular systemic circulation, where its circulation will be severely curtailed within biological systems from its increased size.

The benefits and advantages offered by both aforementioned classes of materials could be brought together under one systematic purview via the formation of composites. Composites, by definition, constitute two or more significantly different constituents, with the resulting properties differing significantly from either [39]. Combining the malleability, ease of processability and scalability of polymers[40] and the porosity and accessibility of hybrid inorganic-organic materials $[31,41]$ makes for a unique composite, exhibiting the adaptability and durability of polymeric structures [42], and the enhanced porosity [43], high surface areas [44], and multi-functionality [45] of hybrid inorganic-organic materials, all within one continuous structure [46].

The study of MOF-polymer composites [46-48] in its many forms has surged in the past two decades due to increased interest in energy and environmental implications of MOFs. However, the majority of these aforementioned studies are focused on the improvement and enhancement of the performance of the resulting nanocomposites as 
opposed to actually determining the intricacies related to the dynamic molecular interactions between the MOFs and its respective polymer matrices. A systematic study involving the influence and interactions of the mechanical properties of MOF nanoparticles (framework rigidity, flexibility, compressibility etc.) [49] and the polymer matrices (glassy, rubbery) are relatively scarce in the current literature [50-53]. In this work, we are presenting a detailed characterisation to obtain dynamic molecular interactions underpinning a polyurethane (PU)/ZIF-8 nanocomposite system, pertaining to its thermo-mechanical and viscoelastic responses. This study is also unique in that the structure-property relationship or the performance study of this particular combination of $\mathrm{MOF} /$ polymer has hitherto not been reported, and although their respective structural analogues were [54, 55], most are limited to chemical synthesis and non-mechanical performance studies. The correlation established in this work will also serve as a model for other rubbery polymer/MOF composites, allowing us to elucidate and predict their respective responses within a single system and pinpoint suitable applications vis-à-vis established parameters. 


\section{Materials and Methods}

\subsection{Synthesis of the nanoparticles (Zeolitic Imidazolate Framework: ZIF-8)}

Nanoparticles of ZIF-8 [ $\mathrm{Zn}(\mathrm{mIm})_{2} ; \mathrm{mIm}=2$-methylimidazolate] [20], as shown in Fig. 1(a), were prepared in accordance to the previously reported room-temperature rapid synthesis method $[50,56]$. However, in this work, the colloidal suspension of ZIF-8 was conducted in tetrahydrofuran (THF).
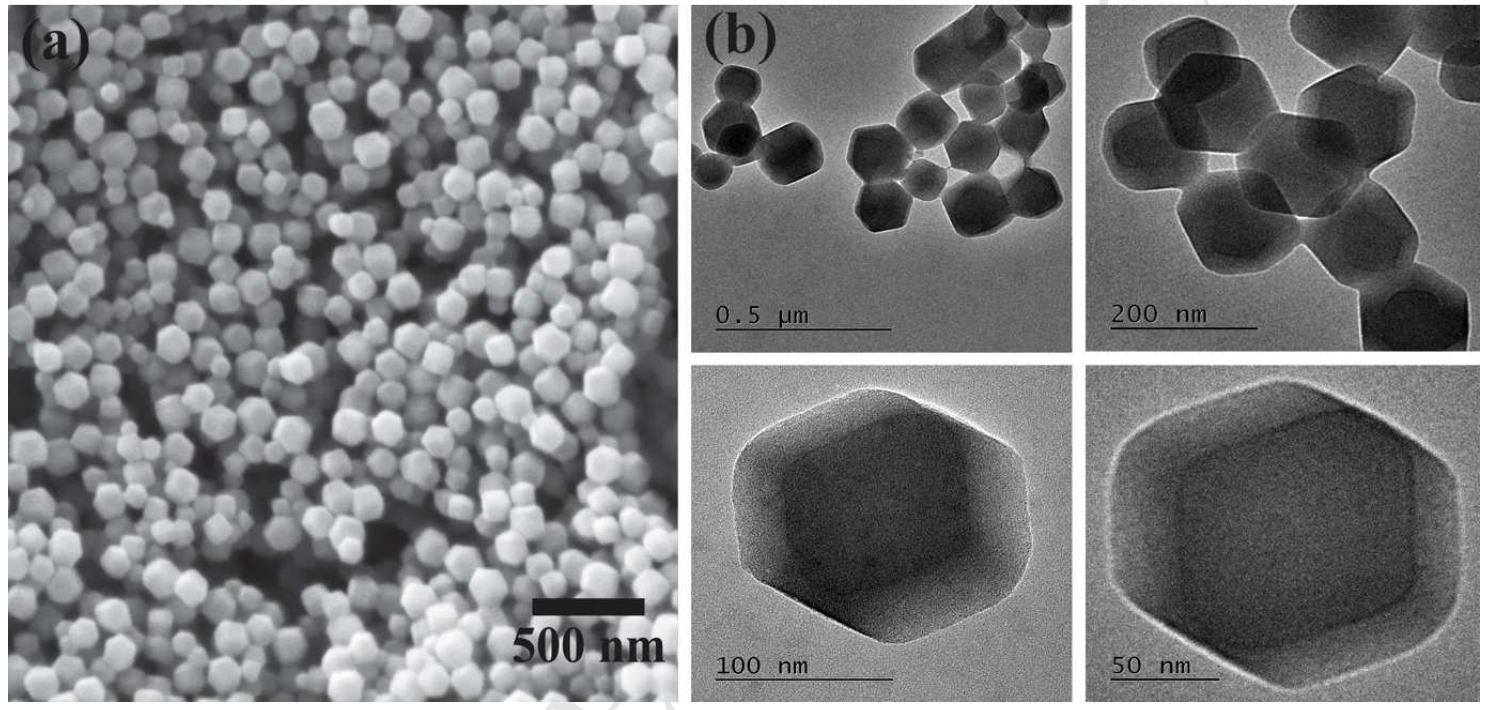

Fig. 1 The synthesised ZIF-8 nanoparticles, with (a) FEG-SEM images taken at $\sim 30,000 \times$ magnification, and (b) TEM images taken at $~ 80,000-120,000 \times$ magnification. The particle size was determined to be $147.26 \pm 7.05 \mathrm{~nm}$ [50].

\subsection{Fabrication of Polyurethane (PU)/ZIF-8 Nanocomposites}

The Polyurethane (PU) polymer solution was prepared by dissolving Poly [4,4'-methylenebis(phenyl isocyanate)-alt-1,4-butanediol/di(propylene glycol)/polyca prolactone] beads (purchased from Sigma Aldrich and used as is without further alterations) in THF for a total of 24-48 hours, until the pellets were visually determined to have completely dissolved in the THF-laden solution. Fig. 2 details the chemical structure(s) of the PU molecular building blocks. 


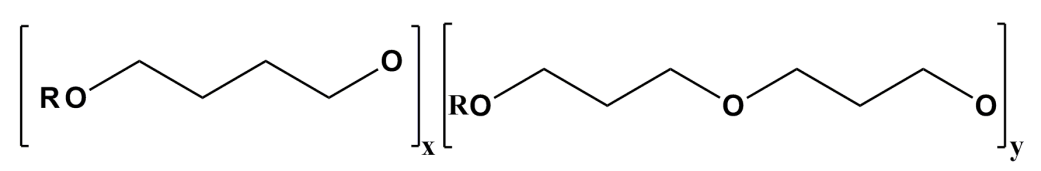<smiles>[R]OCCCCCCC(=O)OC</smiles>

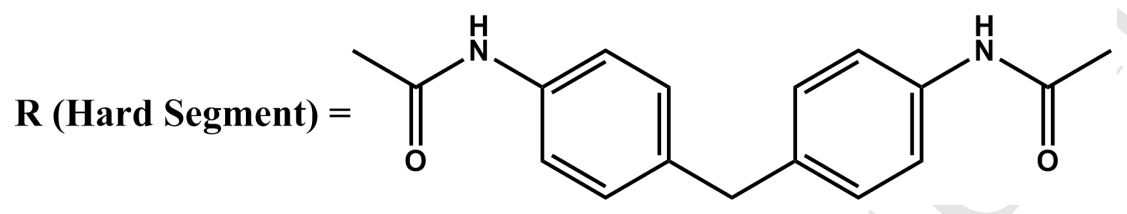

Fig. 2 The chemical structure of the PU used in this work. The hard segment (marked $\mathbf{R}$ in the main structure) could form secondary chemical bonds with the ZIF-8 nanoparticles (see Fig. 10).

Subsequently, the previously synthesised ZIF-8 nanoparticles dispersed as colloids were added in stages to the PU solution, corresponding to the wt.\% equation below (Eqn. (1)):

$$
\mathrm{ZIF}-8 w t . \%=\left(\frac{m_{\text {ZIF }-8}}{m_{\text {ZIF-8 }}+m_{P U}}\right) \times 100 \%
$$

where $m_{\mathrm{ZIF}-8}$ is the weight of the ZIF- 8 nanoparticles dispersed in THF, and $m_{P U}$ is the weight of the PU beads dissolved in THF. This results in the production of four distinct samples of nanocomposites, featuring varied ZIF-8 loadings (Fig. 2): 10, 20, 30, and 40 wt.\%, respectively. Importantly, Song et al. [57] confirmed that the application of this colloidal solution mixing approach may significantly reduce the danger of nanoparticle agglomeration, otherwise witnessed in nanocomposites produced by re-dispersion of dried ZIF-8 nanoparticles [52].

To create the nanocomposites depicted in Fig. 3, the PU/ZIF-8 solutions were cast using the doctor-blade method at a speed of $15 \mathrm{~mm} \mathrm{~s}^{-1}$ onto a PTFE substrate, resulting in an average membrane thickness of $\sim 100 \mu \mathrm{m}$ for each nanocomposite (Fig. 
S1 in SI). The resultant nanocomposites were then transferred to a glove bag saturated with THF vapour, and left to slow-cure for 24 hours, after which they were removed from the glove bag and placed in a vacuum oven, and dried for an additional 24 hours at $85{ }^{\circ} \mathrm{C}$ to remove any remaining occluded THF solvents. After 24 hours, the nanocomposites were detached from the PTFE substrate via submersion in water, and stored for subsequent studies.

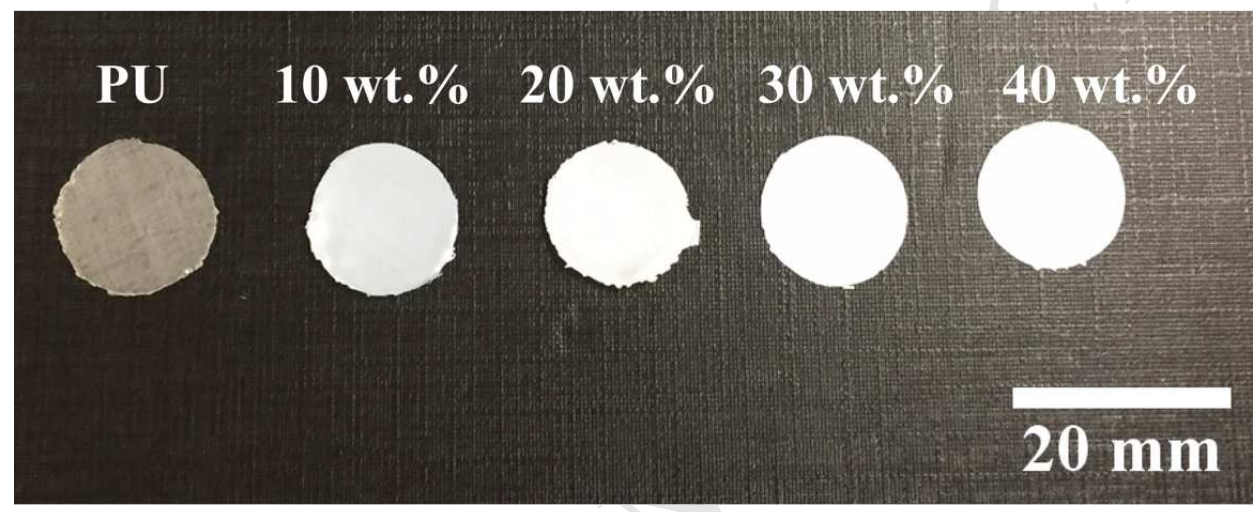

Fig. 3 The PU/ZIF8 nanocomposite membranes. Notice the increased in opacity of the nanocomposites with increasing ZIF-8 nanoparticle wt.\% loading.

\subsection{Materials Characterisation}

The following characterisation methods were chosen for their respective capabilities to determine the morphological, thermo-mechanical, and viscoelastic properties of the PU/ZIF-8 nanocomposites.

Due to its rubbery nature, the PU and its corresponding nanocomposites were freeze-fractured in a liquid nitrogen $\left(\mathrm{LN}_{2}\right)$ bath to expose its internal morphology for imaging purposes. The samples were then mounted at a $90^{\circ}$ angle on a scanning electron microscope (SEM) stub, and were coated with gold in a sputter chamber for 30 secs. The samples were then imaged using the SEM (TESCAN LYRA3 FEG- 
SEM/FIB apparatus), at $5 \mathrm{kV}$ under high vacuum and magnifications of 10,000$30,000 \times$.

The thermal stability and decomposition of the nanocomposites was investigated using the TGA-Q50 (TA Instruments), equipped with an induction heater (maximum temperature of $1000^{\circ} \mathrm{C}$ ) and a platinum sample holder. Samples weighing an average of $4 \mathrm{mg}$ were loaded onto the sample holder and the samples were heated from $50-1000{ }^{\circ} \mathrm{C}$ at a rate of $10{ }^{\circ} \mathrm{C} \mathrm{min}^{-1}$, to the point of complete decomposition. The data was collected and plotted using TA Analyser, which is TA Instrument's propriety software.

\subsection{Thermo-mechanical properties characterisation}

Temperature-dependent viscoelastic measurements were performed using the TA Instruments Q800 Dynamic Mechanical Analyser (DMA) equipped with an $\mathrm{LN}_{2}$ attachment. The dynamic experiments were performed at a heating rate of $3.5^{\circ} \mathrm{C} \mathrm{min}{ }^{-1}$ from -50 to $200{ }^{\circ} \mathrm{C}$. The DMA was calibrated to a pair of tension film clamps and fixed to a gauge length of $\sim 12.5 \mathrm{~mm}$, with all samples tested under uniaxial tensile mode. The static force was set at $0.1 \mathrm{~N}$, with the force track set to $125 \%$. The multifrequency sweep setting was applied, where the oscillating frequency was cyclically alternated between $2,5,10,15,20$, and $25,30,35$, and $40 \mathrm{~Hz}$ as the temperature was increased throughout the experiments.

The approach in determining the quasi-static nanomechanical properties were reported elsewhere and replicated in this work [50]. The Poisson's ratio in this study is assumed to be $\sim 0.5$ (at compression strain of less than $~ 5 \%$ ) [58], indicating near incompressibility typical of a rubbery polymer material.

The stress-strain plots were generated using the Instron universal testing machine (Model 5582), equipped with a $100 \mathrm{~N}$ load cell. The width and gauge length 
of the test coupons were $5 \mathrm{~mm}$ and $30 \mathrm{~mm}$, respectively (refer to Fig. S1 for relative thicknesses of $\sim 100 \mu \mathrm{m}$ ). The samples were clamped to the rig, and were subjected to a tensile load applied at displacement rate of $10 \mathrm{~mm} \mathrm{~min}^{-1}$ until sample failure. The load-displacement plots were converted to nominal stress-strain plots using Digital Image Correlation (DIC), where images of speckled test coupons undergoing tensile deformation were taken at $5 \mathrm{fps}$, and these images were later processed using DaVis ${ }^{\circledR}$ software to calculate the uniaxial strain profile of the test coupons from the previously captured images. These strain profiles were then matched to the stress values calculated from the gauge length and cross sectional area using MATLAB $^{\circledR}$ to produce a nominal stress-strain plot for the PU/ZIF-8 nanocomposites. The generated stressstrain profile were also fitted using the Ogden model [59] via Abaqus ${ }^{\circledR}$, employing its built-in hyperelastic function fitting module.

\section{Results and Discussion}

Fig. 4 shows the cross-sectional images of the PU/ZIF-8 nanocomposites. The dispersion of the nanoparticles within the matrix appears to be uniform, although at lower wt.\% loadings, they tend to form dispersed clusters within the matrix, as opposed to being dispersed as individual, isolated nanoparticles [60, 61]. However, at higher loadings, this trend is supplanted by the more uniform and regular dispersion of the nanoparticles everywhere throughout the matrix. It is also evident that the majority of ZIF-8 nanoparticles retained its original configuration (rhombic dodecahedron) [62] even within the PU matrix, suggesting that the nanoparticles are nominally attached to the rubbery polymeric chains of the PU as opposed to being encapsulated/embedded within the matrix. Also, agglomeration and aggregation do not seem to be an issue with these nanocomposites, as evidenced in Fig. 4. 

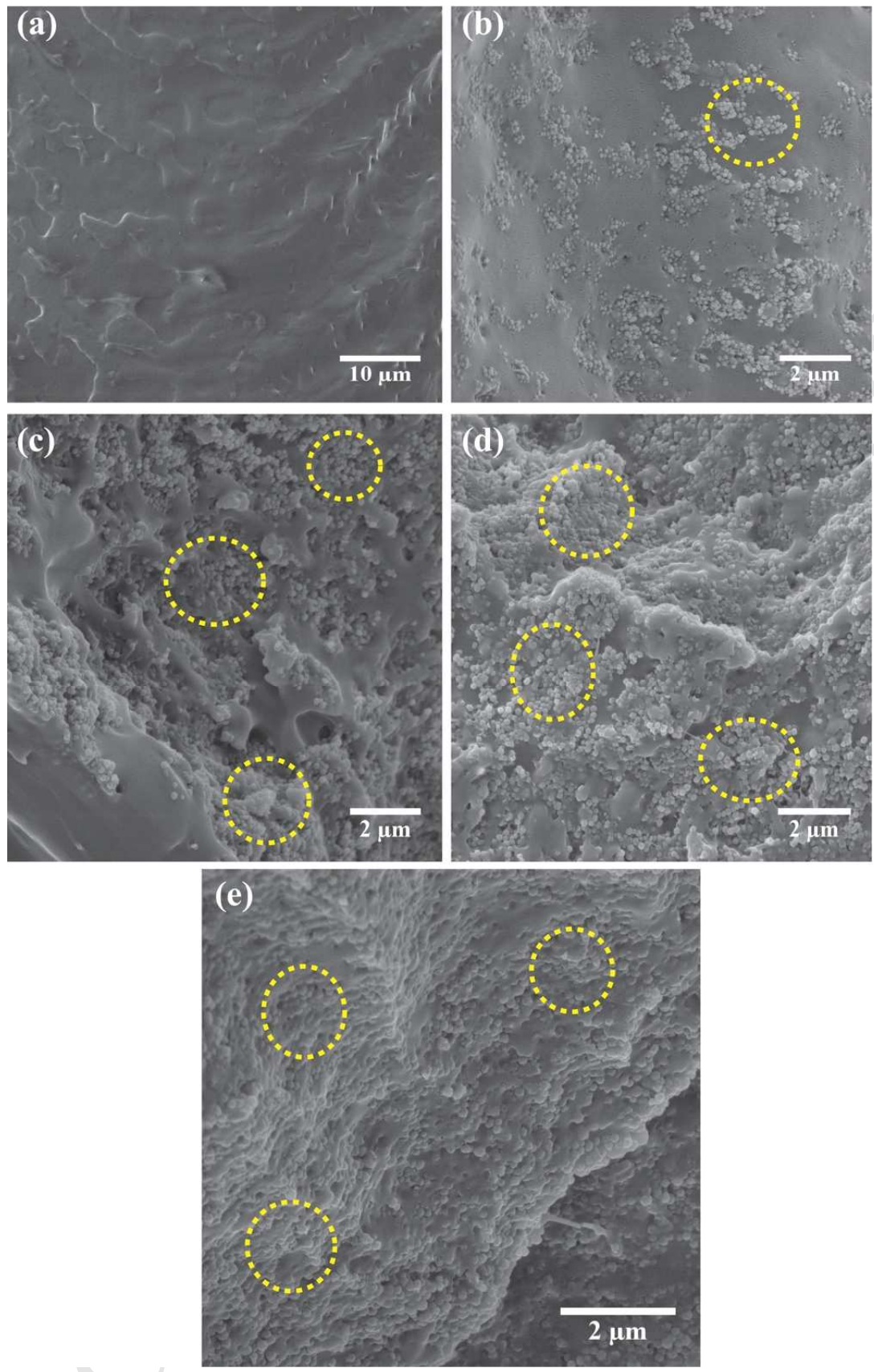

Fig. 4 Cross-sectional FEG-SEM images of PU/ZIF-8 nanocomposites, with (a) PU, (b) 10 wt. $\%$, (c) 20 wt.\%, (d) 30 wt.\%, and (e) 40 wt.\% ZIF-8 nanoparticles, at $30,000 \times$ magnification. The yellow markers are used to highlight the presence of ZIF-8 nanoparticles, and it is quite evident that they form a distinct and distinguishable phase from the PU matrix.

The microstructures suggest that there is a lack of dedicated interfacial layers between the PU primary (hard) and secondary (soft) polymeric chain (Fig. 2) and ZIF- 
8 nanoparticles, and that the interactions are limited to nanoparticle surface interactions, which are associated with weak molecular interactions such as vdW forces, $\pi-\pi$ stacking, and H-bonds $[63,64]$ with the 'hard' group of the PU. Due to the fact that a spherical configuration is thermodynamically favourable [65] and the wellknown tendency for nanoparticles to form agglomerates/aggregates [66], it is reasoned that instead of disrupting the polymeric chain packing of $\mathrm{PU}$, the ZIF-8 nanoparticles attached themselves to the polymeric molecular chains in a manner similar to secondary groups, or in some cases, form new distinct (relatively shorter) chains (in the form of clusters) altogether within the matrix, resulting in new molecular chains of ZIF-8 or combined molecular chains of PU and ZIF-8. In fact, this interaction is evident in the PXRD scans (Fig. S2 in SI), showing distinctive and identifiable peaks of ZIF-8 against an amorphous background of the PU matrix; at room temperature, PU is rubbery and amorphous, therefore, its molecular chains are free to slide and reconfigure themselves when subjected to external stimuli such as thermal energy (heat) or mechanical stress/strain [67-69].

We propose that the introduction of ZIF-8 nanoparticles will only induce (minimal) rearrangement/reformation of the polymeric molecular chain without interrupting the inter- and intra-chain interactions between PU primary and secondary molecular chains, thus allowing the newly formed nanocomposites to retain to its rubbery configuration despite the introduction of filler phases (herein ZIF-8 nanoparticles) within the matrix. However, at increased filler loadings, as seen in Fig. 4 (d)-(e), the presence of ZIF-8 nanoparticles began to underpin and prevent free slippage and movements of PU polymeric molecular chains via interactions between the mIm of ZIF-8 and PU molecular primary chains, stiffening the usually compliant PU matrix $[70,71]$. This is believed to be the function of quantity; higher loadings of 
ZIF-8 nanoparticles meant increased amounts of ZIF-8 nanoparticles per volume within the nanocomposites, resulting in more and more PU molecular chains being pinned and becoming immobilised. The quantification of this molecular alteration and its qualitative effects upon the structural behaviour of nanocomposites will be discussed and detailed in $\S 3.2,3.5$, and 3.6 of this work via quasi-static and thermomechanical characterisation approaches.

\subsection{Nanoindentation Studies}

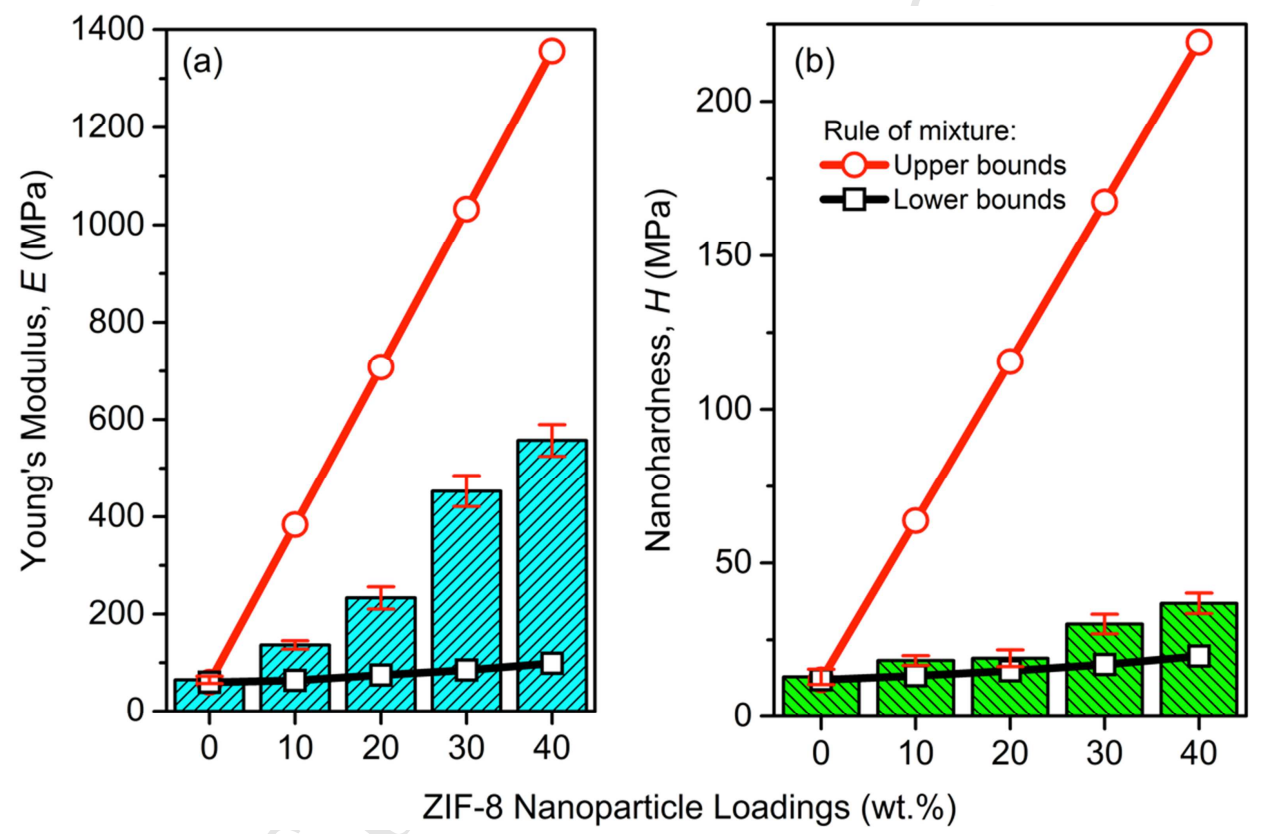

Fig. 5 The compilation of the nanoindentation data determined from the PU and PU/ZIF-8 nanocomposites, with (a) Young's modulus $(E)$ and (b) nanohardness $(H)$. The results are the average of a total of 45 indents from 3 samples upon a surface penetration depth of $2 \mu \mathrm{m}$. The theoretical values are determined using the simple rule of mixture, $E_{\mathrm{UB}}=\varphi_{\mathrm{ZIF}-8} \cdot E_{\mathrm{ZIF}-8}+\left(1-\varphi_{\mathrm{ZIF}-8}\right) \cdot E_{\mathrm{PU}}$ and $E_{\mathrm{LB}}=\left[\left(\varphi_{\mathrm{ZIF}-8} / E_{\mathrm{ZIF}-8}\right)+\left(1-\varphi_{\mathrm{ZIF}-8}\right) / E_{\mathrm{PU}}\right]^{-1}$, where $E_{\mathrm{ZIF}-8}=3.3 \mathrm{GPa}[72]$ and $H_{\mathrm{ZIF}-8}=500 \mathrm{MPa}$ [73], and $E_{\mathrm{PU}}=65 \mathrm{MPa}$ and $H_{\mathrm{PU}}=$ $15 \mathrm{MPa}$ (established from measured PU samples at $0 \mathrm{wt} . \%$ ). Notably, the major overestimation from the upper bound (UB) model indicates that the intra-molecular interface between the ZIF-8 filler phase and the PU matrix is particularly weak, such that there has been a significant reduction in load transfer compared with the idealised (UB) equal strain model [74]. 
Fig. 5 summarises the results from the nanoindentation studies of the PU/ZIF-8 nanocomposite. The Young's modulus $(E)$ and nanohardness $(H)$ of the PU/ZIF-8 nanocomposites were calculated by assuming an isotropic response within the area being indented and using the load-displacement data derived from the indentation tests (Fig. S5-S7).

The results indicate that the $E$ and $H$ are directly proportional to ZIF-8 nanoparticle loadings (wt.\%). As a matter of fact, the increase of both values is quite significant vis-à-vis the loading for both $E$ and $H$. This is evident from looking at PU (0 wt.\%) to PU/ZIF-8 (10 wt. $\%$ ), where the $E$ and $H$ value increased from $\sim 65 \mathrm{MPa}$ to $\sim 137 \mathrm{MPa}$ (increase of $\sim 110 \%$ ) and $\sim 13 \mathrm{MPa}$ to $\sim 18 \mathrm{MPa}$ (increase of $\sim 40 \%$ ), respectively, and from PU/ZIF-8 (10 wt.\%) to PU/ZIF-8 (20 wt.\%), where the values increased from 137 MPa to $233 \mathrm{MPa}$ (increase of $\sim 70 \%$ ) and $\sim 18 \mathrm{MPa}$ to $\sim 19 \mathrm{MPa}$ (increase of $\sim 4 \%$ ), respectively. Overall, an increase of over $760 \%$ in $E$ and $185 \%$ in $H$ is observed at peak ZIF-8 loading (40 wt.\%) from neat PU samples, indicating a significant increase in the relative stiffness and hardness of the nanocomposites. It could therefore be surmised, at this points, that the increased ZIF-8 nanoparticle loadings is enhancing mechanical stability of the nanocomposites while simultaneously introducing accessible porosity and active sites [51, 52].

\subsection{Thermal stability analyses}

Thermal stability is crucial towards the applicability of the nanocomposites, as rubbery polymers in general are known to be extremely susceptible to heat [75]. In this case, the matrix (PU), which forms a structural majority, will be greatly affected by the introduction of heat, however, the ZIF-8 nanoparticles is known to be stable up till $\sim 500{ }^{\circ} \mathrm{C}$ [73]. Establishing the thermal stability of these nanocomposite will allow us to predict, and subsequently model, their behaviour when exposed to higher temperatures, which is 
especially crucial if these nanocomposites are to be used in any practical applications [76, 77].

TGA analysis involves the determination of thermal stability of the samples; basically, the samples are heated at a constant rate within a set temperature range, with its subsequent relative weight decrease $(\%)$ recorded as a function of temperature. This results in a thermal decomposition plot, which will allow us to pinpoint the temperatures that are associated with phase changes, loss of solvents, and its outright decomposition, amongst others [78].

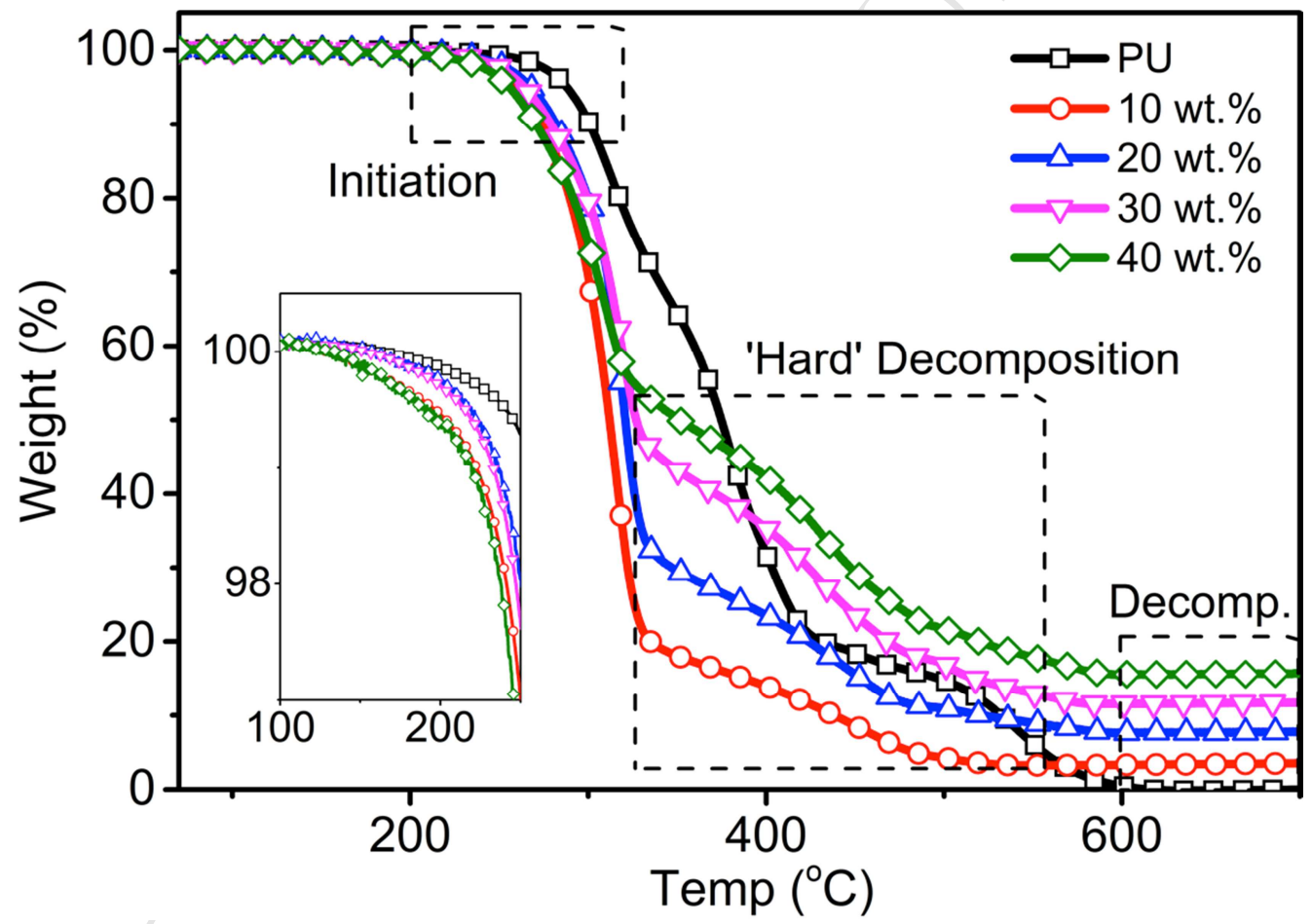

Fig. 6 TGA plots of the PU and PU/ZIF-8 nanocomposites from the room temperature (RT) to $1000^{\circ} \mathrm{C}$. Note the ascending wt. $\%$ as a function of ZIF-8 nanoparticle loadings.

Fig. 6 is the compilation of the results from TGA analysis of the PU/ZIF-8 nanocomposites. All of the samples exhibit similar decomposition trend, which is attributed to the fact that PU forms the structural majority in all of the samples (weight loss exceeding 
$60 \%$ ). The PU sample began decomposing at $250{ }^{\circ} \mathrm{C}$, and continue to do so until $420{ }^{\circ} \mathrm{C}$. At $420{ }^{\circ} \mathrm{C}$, the decomposition process somewhat stabilises, resulting in reduced decomposition rate in the temperature range of 420 to $580{ }^{\circ} \mathrm{C}$, speculated to be the decomposition of the 'hard' segment of the PU (refer to Fig. 2). At temperature T>580 ${ }^{\circ} \mathrm{C}$, the PU sample began to completely decompose. The nanocomposites undergo decomposition initiation at much lower temperatures, and Table S1 (in the SI) summarises the stages and their respective corresponding temperatures. It is generally seen here that the initial, 'hard' segment, and complete decompositions occur a lot sooner for the nanocomposites compared to that of neat PU. However, it should also be pointed out that at higher loadings of ZIF-8 nanoparticles, the rate of decomposition is decreased, where despite earlier initiation temperatures for all stages of decomposition, the weight $\%$ of the samples remaining after undergoing decomposition is a lot higher than its PU counterpart, and this value increases with increasing ZIF-8 nanoparticle loadings. This can be attributed to the interactions between ZIF-8 nanoparticles and the PU matrix; the complete decomposition temperature of PU and ZIF-8 differ significantly, for PU, its $\sim 200{ }^{\circ} \mathrm{C}$ [79], while for ZIF-8, it was reported to be $\sim 500{ }^{\circ} \mathrm{C}$.

Our TGA data suggest that the bonds between the mIm of ZIF-8 and the hard segment of the PU prevented complete structural degradation by maintaining structural integrity via combined weak interactions (H-bonds, vdW forces, and $\pi-\pi$ stacking) with the remaining PU (hard segment) matrix that has yet to be degraded. Only when the ZIF-8 nanoparticles themselves began decomposing (at $\mathrm{T}>500{ }^{\circ} \mathrm{C}$, via the degradation of mIm linkers) that full decomposition takes place. Based on these results, it could be surmised that the integration of ZIF-8 nanoparticles actually helps to enhance the structural-thermal stability of the nanocomposites as a whole. This is also supported by the glass transition $\left(T_{\mathrm{g}}\right)$ determination of the nanocomposites (Fig. S4); the values of the $T_{\mathrm{g}}$ remained close to one another (within $\pm 3{ }^{\circ} \mathrm{C}$ ), confirming that $\mathrm{PU}$ is still dominant vis-à-vis the thermal stability of the 
nanocomposites $[57,80]$. The combined secondary chemical interactions allow the primary and secondary polymeric chains to react to the introduction of external stimuli accordingly, undergoing phase changes and molecular motions in a manner similar to when the nanofillers are absent [57]. In effect, we gathered evidence that the nanofillers ZIF-8 acts in synergy with the PU polymeric chains at $\mathrm{T}>\mathrm{RT}$, thereby strengthening the structural stability of nanocomposite and improving the thermal degradation resistance of the nanocomposite [81].

\subsection{Viscoelastic studies}

Polymeric materials in general, and rubbery polymers in particular, are known to demonstrate viscoelastic characteristics at low oscillatory strains. The study of viscoelasticity makes up the core fundamentals of polymeric materials; this is especially true for rubbery polymers [82]. It is vital that the viscoelastic response of the nanocomposites be determined, due to the structural dominance of PU in the nanocomposites. This will help us analyse the molecular influence of ZIF-8 on the viscoelastic response of PU, and also model and predict the thermo-mechanical response of the subsequent PU/ZIF8 nanocomposites. The structural and chemical changes within the internal configuration of the matrix can be detailed and described accurately using the dynamic modulus ( $E^{*}$, Eqn. [4]), constituting the storage modulus $\left(E^{\prime}\right)$, representing the elastic strain energy stored within the polymeric chains while undergoing oscillatory motions, and the loss modulus ( $E$ "), representing the energy dissipated by the polymer during chain or bond rotations or phase changes [82].

$$
E^{*}=\frac{\sigma^{*}}{\varepsilon^{*}}=E^{\prime}+i E^{\prime \prime} \quad ; \quad \tan \delta=\frac{E^{\prime \prime}}{E^{\prime}}
$$

where $\sigma^{*}$ and $\varepsilon^{*}$ are stress and strain as a function of time, $i=\sqrt{-1}$ is the imaginary component of the loss modulus term $E^{\prime \prime}$, and $\tan \delta$ is the ratio of the storage and loss moduli, often call the dissipation factor. 


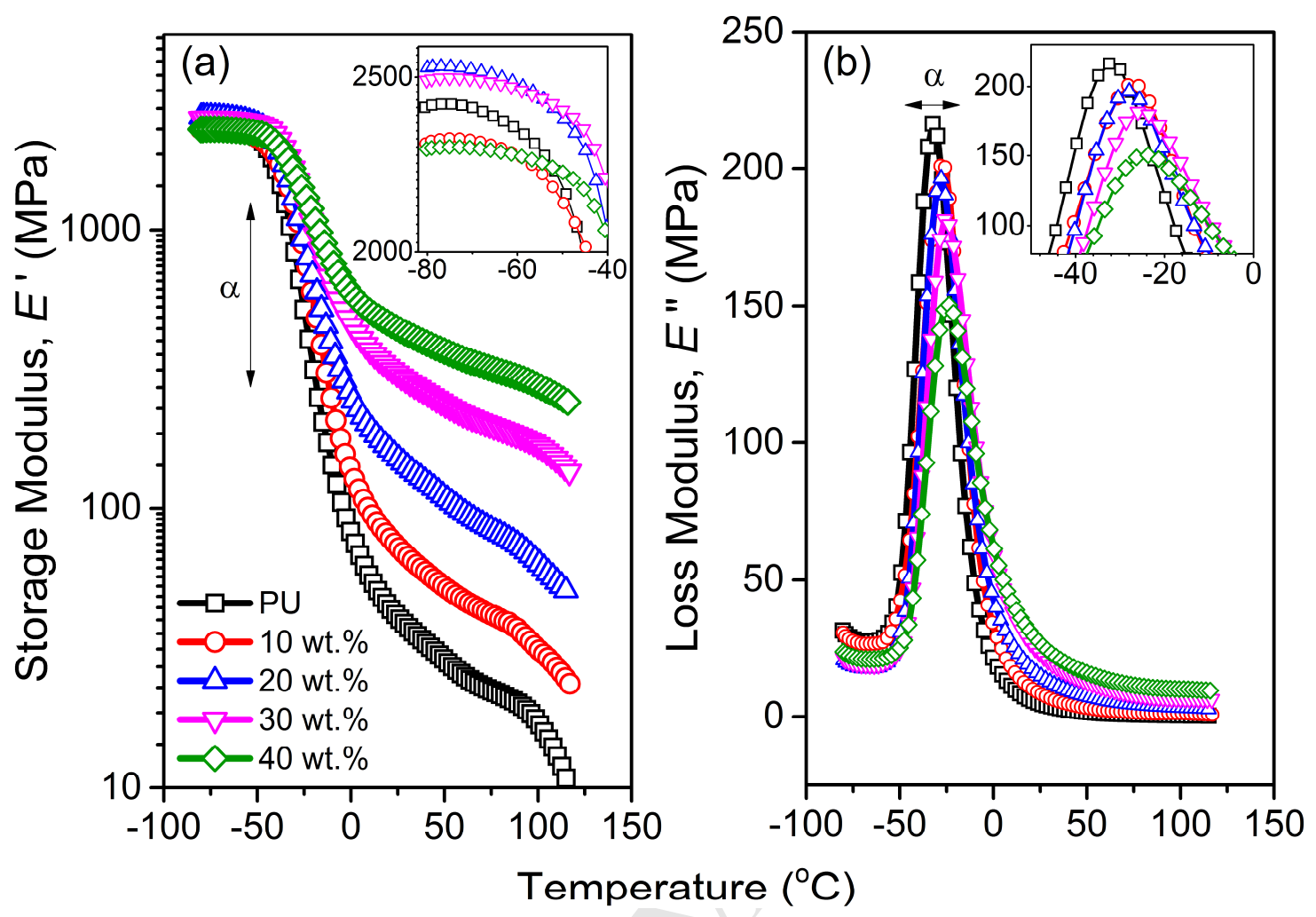

Fig. 7 Representative plots of (a) storage modulus, E' (log-scale) and (b) loss modulus, E", of the PU/ZIF-8 nanocomposites $(f=10 \mathrm{~Hz})$. The insets in both (a) and (b) represent the initial loadings during the experiments, while $\alpha$ denotes the primary relaxation regions of the nanocomposites, which corresponds to the $T_{\mathrm{g}}$ of the nanocomposites, where the primary (hard) molecular chains of the PU and the nanocomposites are experiencing molecular shifts (deformation and modification) simultaneously throughout the nanocomposites.

The study of the viscoelastic properties of the PU-ZIF-8 nanocomposites represents an in-depth study of the molecular interactions between two distinct phases within one structure, due to the fact that polymeric materials undergo multiple phase changes at different temperature levels, the most common of which are 'glassy' and 'rubbery' regions. These phase changes are characterised by the aforementioned molecular interactions; where the glass transition temperature $\left(T_{\mathrm{g}}\right)$ marks the initiation of phase changes. The glassy region is characterised by high stiffness and interlocking bonds, with restricted molecular flows and close interactions between the molecular chains $\left(T<T_{\mathrm{g}}\right)$, while the rubbery region is 
characterised by a major shift in the polymeric primary and secondary chains and the ability of these chains to freely flow and slip past one another when exposed to external stimuli $(T>$ $T_{\mathrm{g}}$ ). Phase changes are termed 'relaxation' and $T_{\mathrm{g}}$ is the temperature where the polymeric chains will undergo major relaxation (also known as the primary relaxation temperature, or $T_{\alpha}$ ), which explains the subsequent major shift in its physical and chemical characteristics.

Fig. 7 shows the (a) storage $\left(E^{\prime}\right)$ and (b) loss (E') moduli of the PU/ZIF-8 nanocomposites. Although $T_{\mathrm{g}}$ remains unaffected by the introduction of ZIF-8 nanoparticles (Fig. S4), it is immediately noticeable that the relaxation modes of the nanocomposites are very much affected by the introduction of ZIF-8 nanoparticles. It is observed that the nanocomposites experience relaxations at relatively higher values of $E$ ' and lower values of $E$ " as the wt.\% of ZIF-8 nanoparticles increases, which is especially evident in the rubbery region $\left(T>T_{\mathrm{g}}\right)$. This is attributed to the increased presence of ZIF-8 nanoparticles within the nanocomposites and the pinning and 'locking' effect the nanoparticles have on the primary and secondary PU polymeric chains; these polymeric chains will become immobilised and have difficulty in undergoing complete relaxation $\left(E^{\prime}, E^{\prime \prime}<10 \mathrm{MPa}\right)$ due to its association with ZIF-8 nanoparticles, which forces the occurrence of relaxation at much higher stiffness levels to accommodate the ZIF-8 nanoparticles, as seen in Fig. 7 (a) and (b).

Increased loadings of ZIF-8 nanoparticles translate into lesser amounts of PU polymeric chains per unit volume in the nanocomposites, which means that the polymeric chains that are loosely associated with the ZIF-8 nanoparticles undergo relaxation at a level that is common for PU. However, the hard segment of the PU is still bounded to the soft PU chains, and the ZIF-8 nanoparticles, and these combined bonds prevents the occurrence of relaxation at levels common to PU. The nanocomposites as a whole still undergo relaxation at higher levels, and it is speculated that at this point, the mechanical response is dominated by the ZIF-8 nanoparticles as opposed to the already relaxed PU polymeric chains. 
Notably the DMA data (Fig.7(a)) demonstrate that ZIF-8-loaded composite is mechanically more resilient that PU, and this is evident in the fact that the nanocomposites, at higher loadings of ZIF-8 nanoparticles, begin to exhibit more robust mechanical response as opposed to neat PU. The ability of the nanocomposites to retain, distributes, and dissipates energy (injected from external stimuli) also improved with the presence of ZIF-8. For instance, we determined that the loss moduli $E$ " peaked at $225 \mathrm{MPa}$ for the PU, while it dropped to $150 \mathrm{MPa}$ for the PU/ZIF-8 $40 \mathrm{wt} . \%$ membrane, corresponding to a decrease of strain energy dissipation by over $33 \%$.

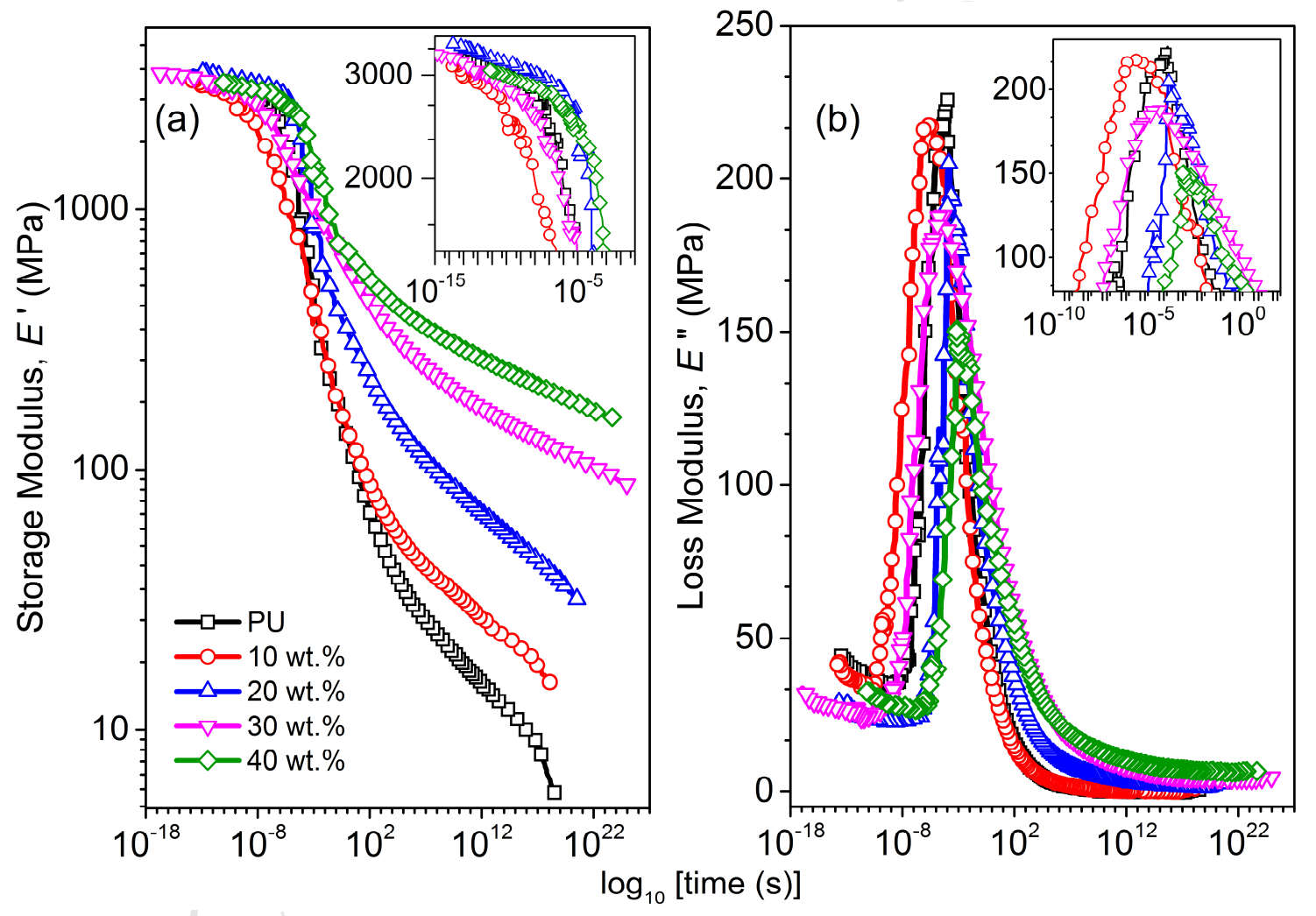

Fig. 8 Time-temperature superposition (TTS) master curves of the PU/ZIF-8 nanocomposites at $T_{\text {ref }}=-15^{\circ} \mathrm{C}$ for the (a) storage ( $\left.E^{\prime}\right)$ and (b) loss ( $\left.E^{\prime \prime}\right)$ moduli, note the log scales for both the $E^{\prime}$ and time $t$. The insets show the earlier time scales for $E^{\prime}$ and $E^{\prime}$. The TTS master curves allow us to access frequency $(f)$ and time $(t)$ ranges that are beyond the capabilities of the DMA experiments.

We assume in this case, as per literature, that PU is thermorheologically simple [83, 84]. Another assumption being made here is that the thermo-mechanical behaviour of the 
PU/ZIF-8 nanocomposites closely mirrors that of neat PU, due to the fact that PU forms the structural majority within the nanocomposites (> $60 \mathrm{wt} . \%)$, and henceforth, the assumption of PU being thermorheologically simple extends to the nanocomposites as well. These assumptions allowed us to construct a corresponding master curve for both the neat PU and the ZIF-8 nanocomposites, and the plots are shown in Figs. 8 (a) \& (b). Generally, at lower temperatures $\left(<T_{g}\right)$ and shorter time spans, the PU and ZIF-8 nanocomposites exhibit lower losses and dissipations while retaining higher energy storage capabilities $\left(E^{\prime}>1,000 \mathrm{MPa}\right.$ and $E "=30 \mathrm{MPa}$ ). The neat PU and the PU/ZIF-8 nanocomposites are physically stiff and exhibit behaviour analogous to glassy polymers $(\sim 3.5 \mathrm{GPa})$. However, at longer time spans and higher temperatures $\left(>T_{g}\right)$, energy retention decreases; this is reflected in the values of $E^{\prime}(500-1,000 \mathrm{MPa})$ and $E^{\prime \prime}(150-230 \mathrm{MPa})$ of the master curve. At this stage, the neat PU and the ZIF-8 nanocomposites exhibit behaviours that are consistent with rubbery polymers. As previously discussed and also seen here, the presence of ZIF-8 nanoparticles decrease the dissipation and increase energy retention by $\sim 30 \%$. Simply put, the introduction of ZIF-8 nanoparticles increases the operational capability of the nanocomposites by dampening the initiation of the complete degradation mechanism induced by constant exposure to heat (-70 to $\left.120^{\circ} \mathrm{C}\right)$ and vibrations $(2,5,10,15,20,25,30,35,40 \mathrm{~Hz})$ upon the nanocomposites. This supposition is also supported by the activation energy $\left(E_{\mathrm{a}}\right)$ of the nanocomposites (Eqn. 5), [85] as shown in Fig. 9.

$$
\ln f=\ln A-\frac{E_{\mathrm{a}}}{R T_{\mathrm{g}}}
$$

where $f$ are the frequencies garnered from the DMA test, $E_{\mathrm{a}}$ is the activation energy (in $\mathrm{kJ} / \mathrm{mol}), R$ is the gas constant $\left(8.3145 \mathrm{~J} \mathrm{~mol}^{-1} \mathrm{~K}^{-1}\right)$ and $T_{\mathrm{g}}$ is the reference temperature (also known as $\left.T_{\text {ref }}\right)$. 


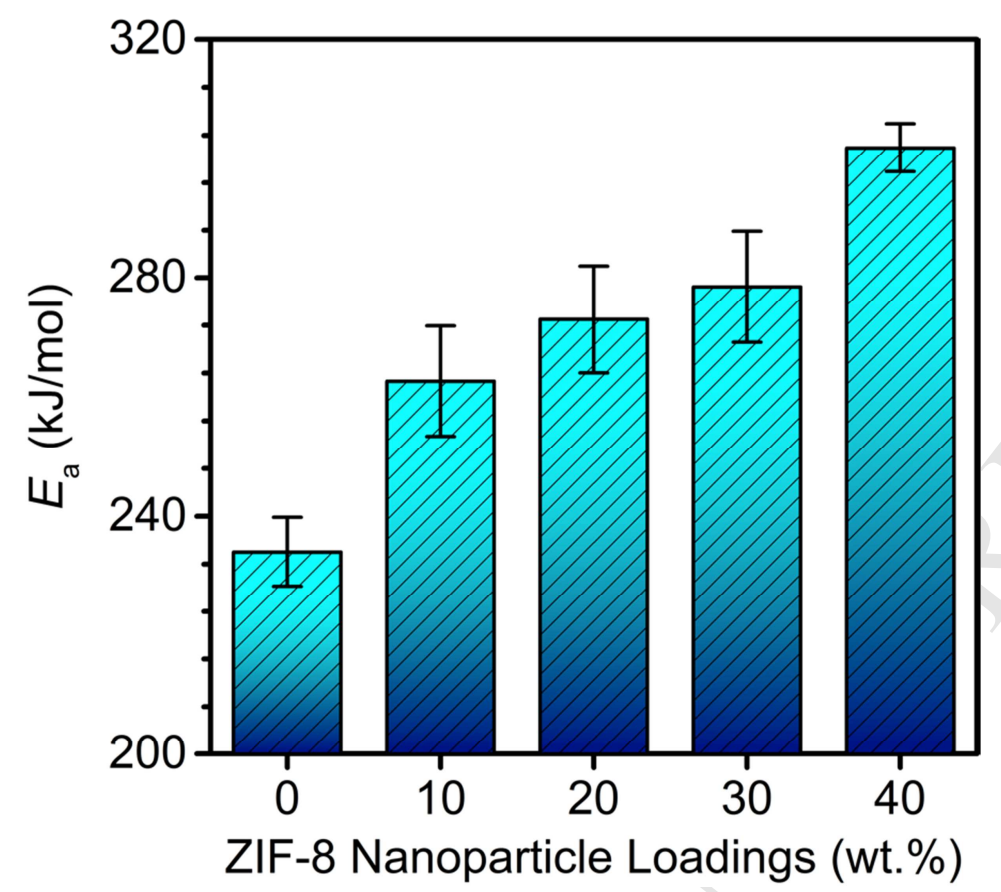

Fig. 9 The activation energy $\left(E_{\mathrm{a}}\right)$ of the PU/ZIF-8 nanocomposites at $T_{\mathrm{g}}$, derived from their respective shift-factor plots based on the Arrhenius model (Fig. S9). Note the upward trend of the $E_{\mathrm{a}}$ as a function of ZIF-8 nanoparticle loadings.

The upward trend of the $E_{\mathrm{a}}$, as evident in Fig. 9, proves that more energy is required to induce a phase change (glassy-to-rubbery) in the nanocomposites, as the amount of ZIF- 8 within the nanocomposites increases. The interaction between the primary and secondary polymeric chains in the PU matrix is rather weak and the polymeric chains are flowing and slipping freely amongst one another, rendering the PU membrane fluid, incompressible, and pliant [86]. As discussed previously, the presence of ZIF-8 nanoparticles will restrict these movements (molecular mobility) and make it harder for free-range motion of polymeric chain via their collective secondary chemical bonds (H-bonds, $\pi$ - $\pi$ stacking, and $\mathrm{vdW}$ forces) between mIm linkers and the hard segment of the PU [87]. This will translate into higher levels of energy needed to induce phase change in the nanocomposites, due to the fact that molecular relaxation is structurally and chemically restricted. The modes of molecular interactions that might exist between the ZIF-8 nanoparticles and the hard segments of the PU are illustrated in Fig. 10. 


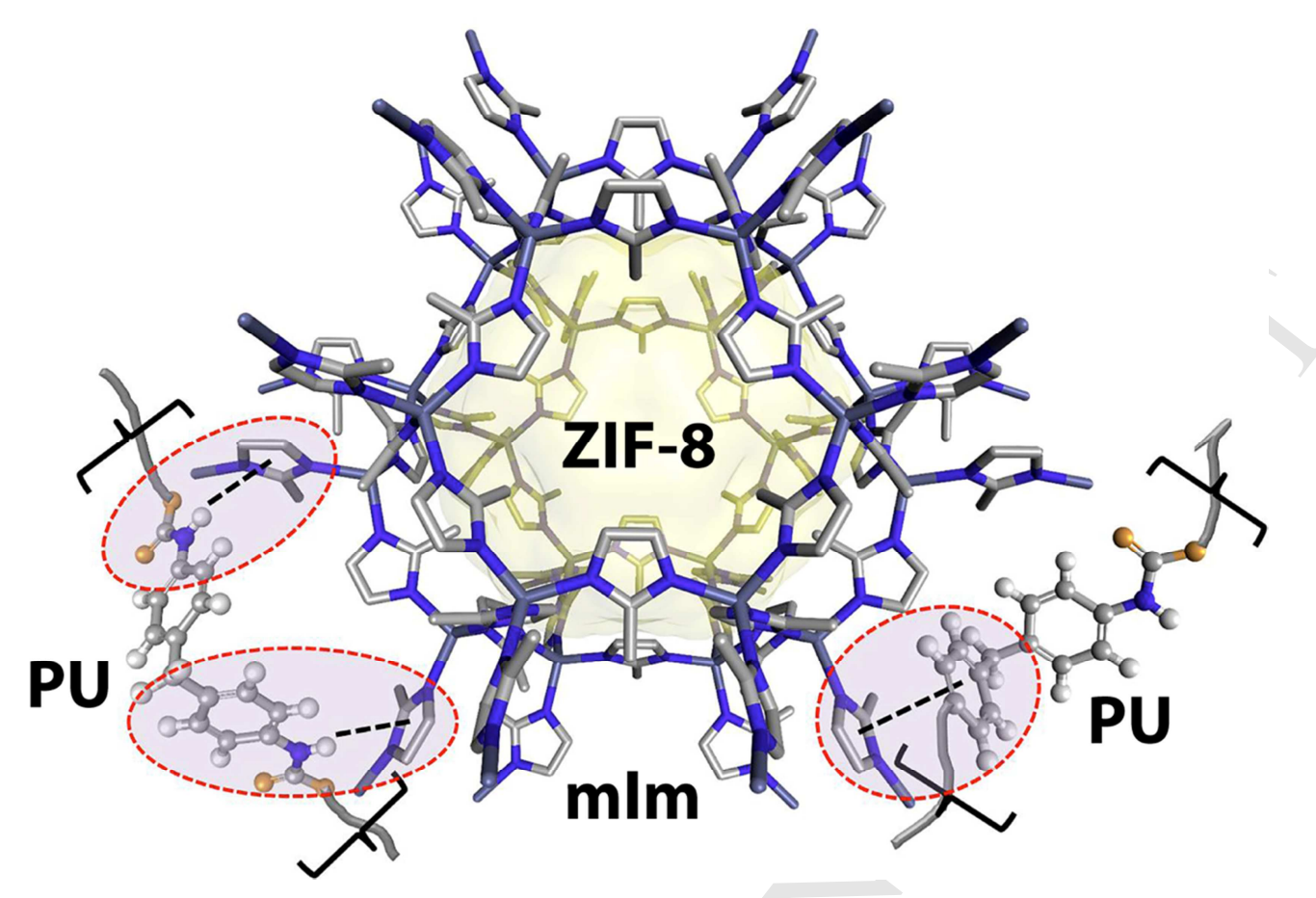

Fig. 10 The chemical interactions between the ZIF-8 nanoparticles (porous framework) and the active ('hard') groups of the PU (labelled as \{\} in the schematics). The bonds being shown in the schematic are hydrogen bonds (between the $\mathrm{N}$ in ZIF-8 and $\mathrm{H}$ in PU, designated as ----), $\pi-\pi$ stacking (N-rings in ZIF-8 and benzene rings in PU, encompassing regions marked with ----- ellipses), and van der Waals (vdW) forces (represented by the colours grey). The strength of these bonds are relatively stronger than the bonds between primary and secondary PU polymeric chains, due to its different constituents ( $\mathrm{N}$ as opposed to $\mathrm{C}$, and the hydrogen of methyl groups), as discussed in Jensen et al. [64] The yellow surface represents the pore accessible volume of the ZIF-8 sodalite cage. Colour scheme adopted: blue - nitrogen, light grey - carbon, dark grey - zinc, gold - oxygen, white - hydrogen.

\subsection{Large strain deformation and mechanical toughness of nanocomposites}

The previous sections dealt with the elastic deformation of the nanocomposites at small strains $(\varepsilon<1 \%)$, which allowed them to retain their original configuration with the cessation of strain loading. For a complete assessments of the thermo-mechanical response of nanocomposites, it is vital that they be tested at extended deformation ranges, preferably up till the point of fracture, which will allow us to analyse the corresponding behavioural responses related to structural failure [52]. We adopted the uniaxial tensile test for this purpose, due to the fact that it is a very well established method for determining the static 
mechanical response of materials towards large strain deformations. Figs. 11 and 12 show the representative nominal stress-strain (henceforth referred to as $\sigma-\varepsilon$ ) plots and the corresponding compilation of mechanical properties derived from the aforementioned plots.

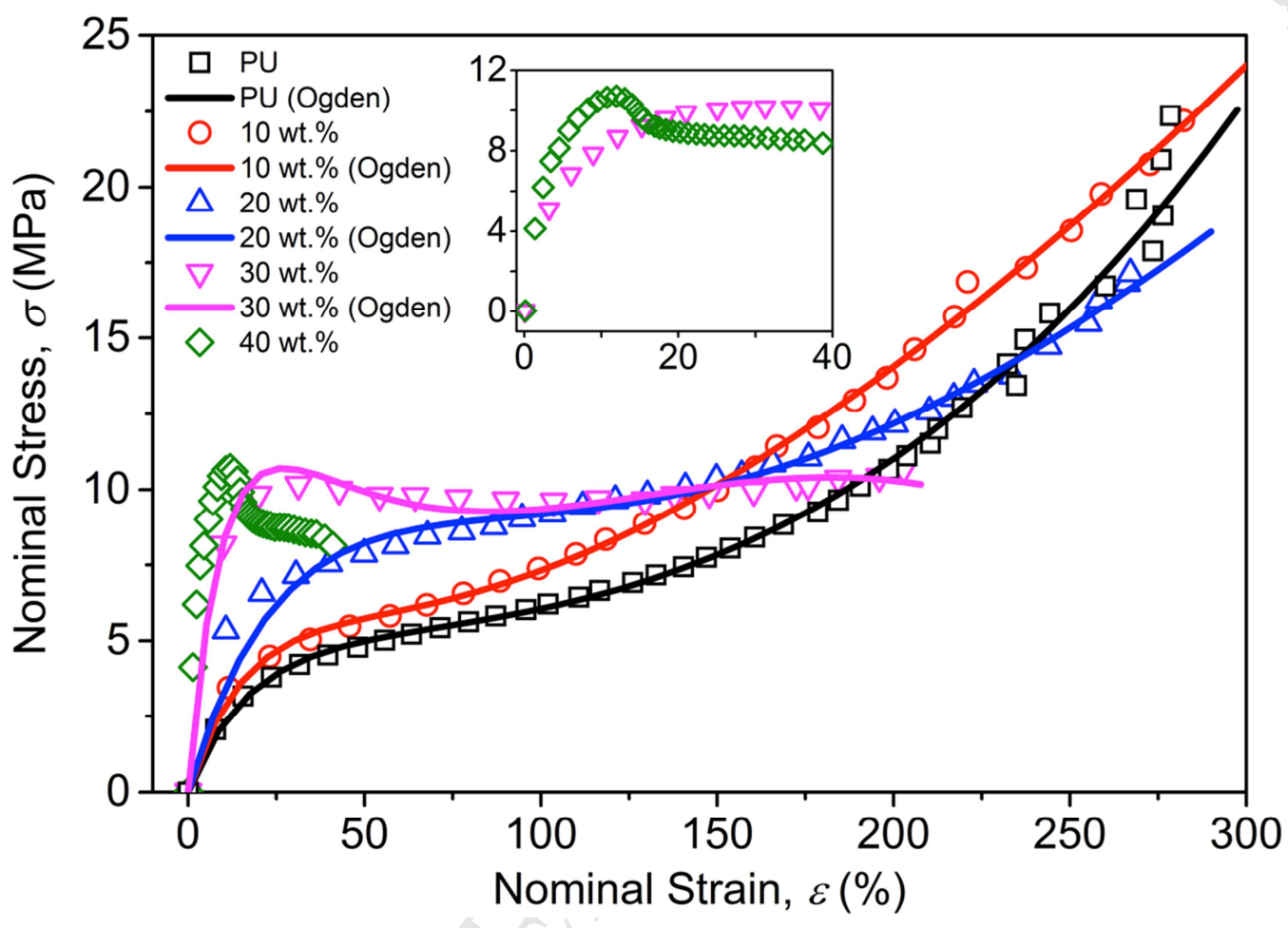

Fig. 11 Representative nominal stress-strain $(\sigma-\varepsilon)$ plots for the PU/ZIF-8 nanocomposites derived from the uniaxial tensile tests and normalised using the Digital Image Correlation (DIC) technique via DaVis ${ }^{\circledR}$. The inset shows the $30 \mathrm{wt} . \%$ and $40 \mathrm{wt} . \%$ nanocomposites, where it is associated characteristics are more evident. The hyperelastic model implemented in the software Abaqus ${ }^{\circledR}$ was used to determine the constants associated with the Ogden model, resulting in a total of 6 material coefficients (Table S2) and fitted to each experimental data set.

The nominal $\sigma-\varepsilon$ plots clearly demonstrated hyperelasticity $(\varepsilon>>100 \%)$ up to the 30 wt.\% membrane, rendering it viable for Ogden-type hyperelastic model fitting given in Eqn. 6 [59]. The subsequent fitting showed the suitability of the Ogden model for these samples, implying that the assumptions (its response to heat is isotropic and its deformation recoverable) associated with the model is also valid. The materials constants associated with the Ogden model are detailed in Table S2 (SI). 


$$
\begin{aligned}
& U=\sum_{i=1}^{N} \frac{2 \mu_{i}}{\alpha_{i}^{2}}\left(\bar{\lambda}_{1}^{\sigma_{i}}+\bar{\lambda}_{2}^{\sigma_{i}}+\bar{\lambda}_{3}^{\sigma_{i}}-3\right)+\sum_{i=1}^{N} \frac{1}{D_{i}}\left(J_{e l}-1\right)^{2 i} \\
& \bar{\lambda}_{i}=J^{-\frac{1}{3}} \lambda_{i} \rightarrow \bar{\lambda}_{1} \bar{\lambda}_{2} \bar{\lambda}_{3}=1
\end{aligned}
$$

where $\mu_{\mathrm{i}}, \lambda_{\mathrm{i}}$ and $\alpha_{\mathrm{i}}$ are material parameters that are determined via model fitting to the experimental data, and $\sigma_{\mathrm{i}}$ is the 3D Cauchy stress associated with the material.
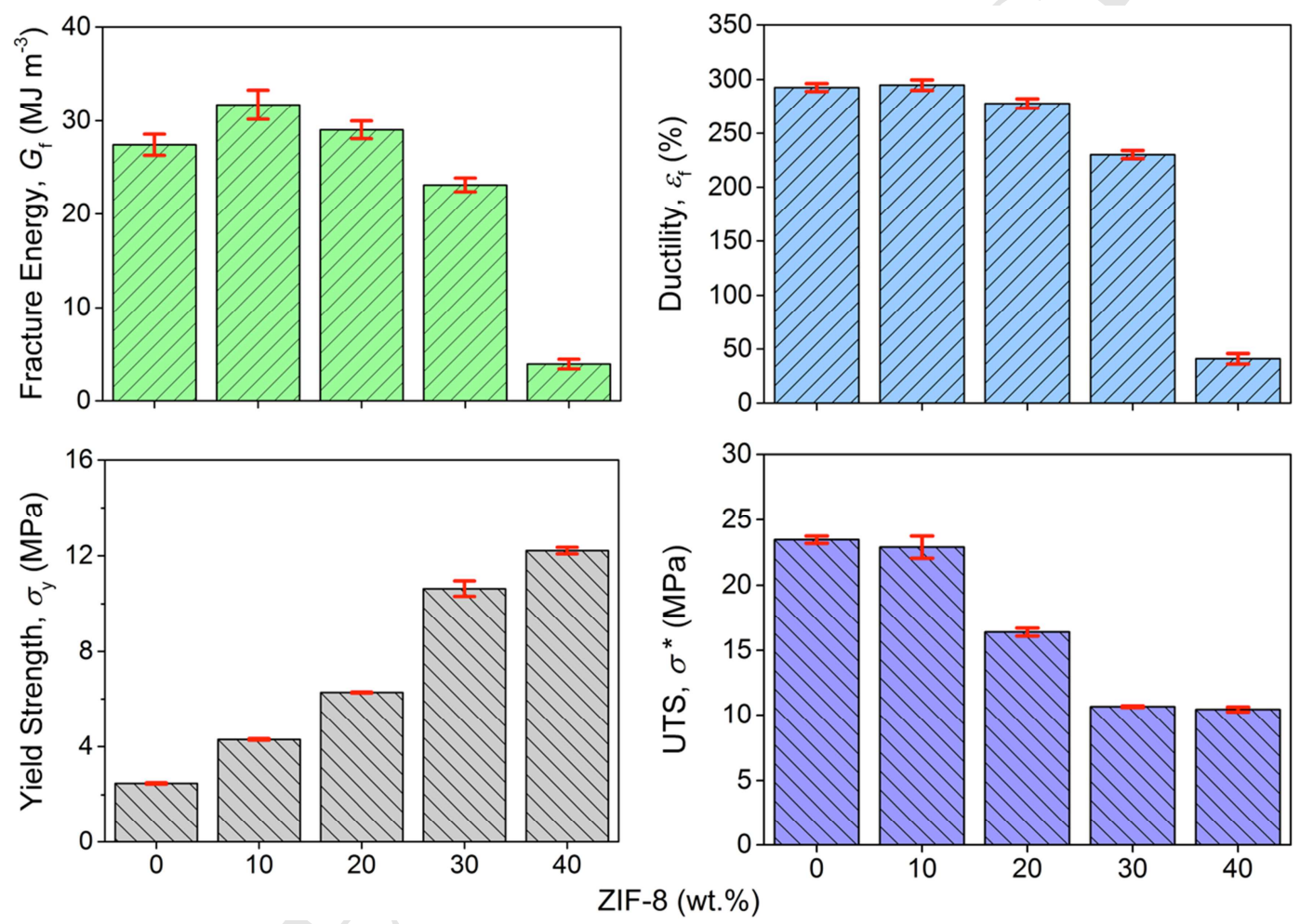

Fig. 12 The mechanical properties of the PU/ZIF-8 nanocomposites derived from the $\sigma-\varepsilon$ plots (Fig. 11), with (a) ductility $\left(\varepsilon_{\mathrm{f}}\right)$, (b) fracture energy $\left(G_{\mathrm{f}}\right)$, (c) ultimate tensile strength $\left(\sigma^{*}\right)$, and (d) yield strength $\left(\sigma_{\mathrm{y}}\right)$. The values were calculated and averaged from a total of three test coupons, and the corresponding standard deviations for each sample are reflected in the error bars.

It is rather obvious that the introduction of ZIF-8 nanoparticles into the PU matrix influence the mechanical properties of the resulting nanocomposites. It is immediately apparent that elongation-to-failure $\left(\varepsilon_{\mathrm{f}}\right)$, fracture energy $\left(G_{\mathrm{f}}\right)$, and ultimate tensile strength $\left(\sigma^{*}\right)$ are all inversely proportional to the addition of ZIF-8 nanoparticles, while the yield strength 
$\left(\sigma_{\mathrm{y}}\right)$ is directly proportional to it (consistent with increase in hardness obtained in Fig. 5(b)). The nanocomposites, up till 30 wt.\%, retain the hyperelasticity inherent in PU (Fig. 11). Substantial and noticeable changes to the nanocomposites are however, more pronounced at loadings exceeding $20 \mathrm{wt} . \%$.

The pinning and nominal immobilisation effect introduced by the ZIF-8 nanoparticles to the PU polymeric molecular chains are not as significant at lower loadings $(<20 \mathrm{wt} . \%)$, due to the scarcity of the particles within the PU matrix. This is also reflected in the FEGSEM images of the PU/ZIF-8 nanocomposites, where the ZIF-8 nanoparticles tend to be evenly dispersed clusters throughout the matrix (Fig. 4 (b) - (c)). However, this changes when the ZIF-8 nanoparticle loadings are higher (> 20 wt.\%), where it is noticed that ZIF-8 nanoparticles begin to dominate the structure and are more pronounced, as evident in Fig. 4 (d) - (e). Pinning and nominal immobilisation begin to dominate the interactions between the ZIF-8 nanoparticles and the polymeric molecular chain of the PU, resulting in loss of hyperelasticity (extended ductility), and with it, fracture energy and tensile strength. With the loss of hyperelasticity, the nanocomposites become stiffer and less stretchable (more brittle), resulting in higher yield strengths, as shown in Fig. 12 (d). It should also be pointed out that the Young's moduli $(E)$ and nanohardness $\left(H\right.$ scales with yield strength $\left.\sigma_{\mathrm{y}}\right)$, as measured by the nanoindentation technique, is directly proportional to ZIF-8 nanoparticles loading, further lending credence to this supposition. All in all, the results from nanoindentation and uniaxial tensile tests are in excellent agreement, while the chemical interaction model being proposed in Fig. 10 can be used to explain the pinning and immobilisation effects introduced by the ZIF-8 nanoparticles (filler phase). 


\subsection{General practical implications of this study}

MOFs, especially ZIFs, are well known for their gas separation capabilities, due to tuneable pores, high functional surface areas, and low densities. As a matter of fact, the majority of work involving ZIFs or nanocomposites of ZIFs focused on its gas separation properties $[52,57]$. However, practical issues, such as its fragility and low scalability prevented the deployment of these MOFs in commercial industrial settings, and as such, reported work involving these nanocomposites are mostly limited to laboratory-scale setups. Although successful at this scale, there are currently not many work involving the MOF or MOF-based nanocomposites for gas separation at scales beyond the lab-scale setup [88]. In fact there is a wide distinction in literature between the functional performances of MOF or MOF-based nanocomposites and its corresponding engineering design, development, and optimisation to afford practical use $[89,90]$. It is fairly evident that these aspects of the nanocomposites are neglected in most studies involving MOF-polymer nanocomposites in literature, with only a few reported examples, such as Cohen et al. [51], discussing the formation of in situ MOF-(ZIF-8, MIL-101, HKUST-1, and UiO-66) PVDF composites for dye separation, resulting in effective but brittle composites, and Feijani et al. [91], who discussed the formation of MOF-(CuBTC, MIL-53, NH2-MIL) PVDF composites for gas separation performance, also reporting similar results alongside poor mechanical properties.

The current work intends to focus on the development and optimisation of the thermomechanical properties and resilience of PU/ZIF-8 nanocomposites. This particular composite has yet to be reported in literature, and this work represent the first attempt in characterising and optimising its design. Furthermore, the combination of a hyperelastic matrix (PU) and a structurally flexible MOF (ZIF-8) [49],[72] represent an interesting opposing mixture, which resulted in unique and distinctive thermo-mechanical and viscoelastic properties. Our supposition and discoveries will be summarised and detailed in the following manner. 
We confirmed that ZIF-8 and PU are linked via tenable collection of secondary interactions and remained semi-independent of each other at lower loadings of ZIF-8 nanoparticles (Fig. 4). Unlike glassy polymer matrices (e.g. Matrimid) [50, 57], ZIF-8 nanoparticles are nominally attached to the primary polymeric molecular chain of PU, as posited in Fig. 10. This basically implies that the free volume and ZIF-8 nanoparticles are not fixed, they are assumed to be a function of external stimuli; the polymeric chain of PU will deform and elongate as per stimuli such as temperature and pressure, and the location of free volume within the rubbery polymer itself will be shifted based on the motion of the polymeric chain. The ZIF-8 nanoparticles, which are nominally attached to the polymeric chains of PU, will shift alongside these motions, or will be debonded from the polymeric chain, forming independent clusters of ZIF-8 nanoparticles not-attached to the PU matrix. However, at higher loadings of ZIF-8, the nanocomposites, despite retaining a respectable elongation-to-failure (> 40\%) and structural integrity, is now quickly becoming dominated by the combined secondary bonds formed between mIm (organic ligands) of ZIF- 8 and the hard segments of the PU, introducing the 'locking' effect discussed previously, resulting in fixed free volume and ZIF-8 nanoparticles, and polymeric chains that are not as free to deform or elongate compared with their lower loaded counterparts. This notion of filler-tomatrix pinning effects is supported by the large strain mechanical deformation data presented in Fig. 12.

Up till now, the formation of free volume and placement of nanofillers in polymer nancomposites are speculated to be random occurrence, however, the phenomenon observed with these robust PU/ZIF-8 nanocomposites opens up an avenue that might lead into bespoke free volume and nanofillers within nanocomposites post-fabrication instead of prefabrication. This would especially be salient to the realm of certain applications (gas 
separations, desalination), as this would also mean that the mechanical properties are tuneable and thermal properties are customisable as well [92].

We demonstrated that the PU/ZIF-8 nanocomposites are able to withstand significant deformation before failing; this is attributed to the hyperelastic nature of the PU matrix. Energy and heat, at moderate levels, are evenly distributed and dissipated throughout the nanocomposites via a combination of properties, such as viscoelasticity (elastic region; $\varepsilon<$ $1 \%)$ and hyperelasticity $(\varepsilon>1 \%)$ [93]. This is evident from the results garnered from DMA and static uniaxial analyses, and discussed in $\S 3.5$ and 3.6, respectively. Under oscillatory elastic strains, the nanocomposites are capable of retaining its original configuration postload removal, at an oscillatory stress of $2 \mathrm{GPa}$. Dissipated energy, in the form of $E$ ", falls between $180-250 \mathrm{MPa}$, implying that only $\sim 10 \%$ of energy is lost per oscillatory cycle, while the remainder is dampened within the nanocomposites. The introduction of ZIF-8 also increased the $E_{\mathrm{a}}$ of phase change (glassy-to-rubbery); the $E_{\mathrm{a}}$ increased by $40 \%$ when loaded at 40 wt.\% of ZIF-8 nanoparticles. This is especially useful, as the $T_{\mathrm{g}}<\mathrm{RT}$, while operationally speaking, $T_{\mathrm{op}}$ would fall between $\mathrm{RT}$ and $100{ }^{\circ} \mathrm{C}$ experienced during gas separation and pervaporation processes.

It was also determined that the addition of ZIF-8 did not significantly affect the macro-mechanical properties of the resulting nanocomposites, where it was seen in Figs. 10 and 11 that the nanocomposites exhibited macro-mechanical properties that are relatively similar to that of neat PU (hyperelastic), such as the fracture energy (a difference of 10\% between PU and PU/ZIF-8 20 wt.\%), elongation-to-failure (a difference of 5\% between PU and PU/ZIF-8 20 wt.\%), and tensile strength (a difference of $15 \%$ between PU and PU/ZIF-8 $20 \mathrm{wt} . \%)$. The relatively small difference in these macro-mechanical properties bodes well for the nanocomposites, as literature had proven that increased content of ZIF-8 nanoparticles greatly increase the performance of nanocomposites in terms of gas separation [57], 
ionic/proton conductivity [47], and pervaporation [94]. By retaining hyperelasticity and the viscoelastic characteristics of the PU matrix despite the addition of ZIF-8 at unprecedented levels, it can be assumed that the performance of these nanocomposites, should it be utilised for the aforementioned applications, would result in significant improvements.

\section{Conclusions}

We present a systematic effort at understanding the thermo-mechanical and viscoelastic properties of a new system of PU/ZIF-8 nanocomposites, which can be readily prepared in the form of $\sim 100-\mu \mathrm{m}$ thick membranes. Despite the lack of this particular grade of nanocomposite in literature, i.e. rubbery polymer matrix combined with MOF nanoparticles (e.g. ZIF-8), the methods used to determine its corresponding properties are quite well established, such as quasi-static nanoindentation and uniaxial tensile tests, and dynamic mechanical analysis (DMA). Our experimental results and detailed analyses lead us to the following conclusions:

- The unique combination of PU (a hyperelastic rubbery polymer) and ZIF-8 (a prototypical zeolitic MOF) allowed the thermal, mechanical, and structural characteristics of both constituents to act in parallel due to the loose association of the constituents, while negating the weaknesses inherent in either. This is evident from the results from TGA, DMA, and uniaxial tensile tests, where the hyperelastic and viscoelastic characteristics of PU was retained despite the introduction of ZIF-8 nanoparticles, which in turn introduced free volume and active porous sites that will be beneficial for applications such as gas capture and separation

- The interactions between the ZIF-8 nanoparticles and PU matrix is tenuous and dominated by a collection of uniform weak interactions, such as H-bonds, vdW forces, and $\pi-\pi$ stacking. Due to the strengths of these bonds, at lower loadings of ZIF-8 
$(<\sim 20 \mathrm{wt} . \%)$, it is anticipated that the free volume and pinning of the ZIF-8 nanoparticles are likely not fixed (thus mobile), and the corresponding size and shape of these free volumes within the nanocomposites are influenced by the introduction of external stimuli from coupled stress and temperature effects. However, at higher loadings of ZIF-8 nanoparticles (> 20 wt.\%), the sheer increase in the amount of weak interactions became quite significant, thereby forming a more sturdy and mechanically rigid nanocomposite, which suppresses the mobility at the ZIF-8/PU interface causing major decline in composite ductility and toughness.

- The methodologies we developed in this study can be extended to other hyperelastic polymers (e.g. PDMS, PVDF, PVP, polypropylene) in combination with a vast range of existing nanostructured MOF-based materials to yield mechanically and thermally robust nanocomposites to enable practical applications.

\section{Supplementary Information (SI):}

ZIF-8 nanoparticles size distribution, membrane thickness distribution, XRD of membranes and crystallinity analysis, nanoindentation load-displacement data, DMA results obtained from multi-strain frequency sweep experiments (multi-frequency), and coefficients associated with the derivation of the Ogden model for hyperelastic materials.

\section{Acknowledgements:}

E.M. Mahdi would like to thank Yayasan Khazanah (YK) for the DPhil scholarship that made this work possible. We acknowledge the Royal Society Research Grants (RG140296) for equipment funding. The authors would like to thank Mr. S. Ying and Prof. A.M. Korsunsky for providing access to the Scanning Electron Microscope facilities (MBLEM Laboratory at Oxford, EU FP7 Project iSTRESS (604646)), and to Prof. Steve Roberts and Dr. David Armstrong for access to the nanoindentation facilities at Oxford Materials. We are grateful to the ISIS Rutherford Appleton Laboratory, especially Dr. Marek Jura and Dr. Gavin Stenning at R53 Materials Characterisation Laboratory, and Dr. James Taylor at R79 Hydrogen and Catalysis 
Laboratory for providing access to XRD and spectrometers. We would like to acknowledge the provision of advanced materials characterisation facilities by the Research Complex at Harwell (RCaH), Rutherford Appleton Laboratory, Oxfordshire. We thank Abhijeet K. Chaudhari for providing the graphics used for preparing Fig. 10.

\section{References}

[1] N.H. Baharuddin, N.M.N. Sulaiman, M.K. Aroua. Removal of Heavy Metal Ions from Mixed Solutions via Polymer-Enhanced Ultrafiltration using Starch as a Water-Soluble Biopolymer, Environ. Prog. Sustain Energy 34 (2015) 359-367.

[2] C. Sprau, F. Buss, M. Wagner, D. Landerer, M. Koppitz, A. Schulz, D. Bahro, W. Schabel, P. Scharfer, A. Colsmann. Highly efficient polymer solar cells cast from non-halogenated xylene/anisaldehyde solution, Energy Environ. Sci. 8 (2015) 2744-2752.

[3] J. Yin, B. Deng. Polymer-matrix nanocomposite membranes for water treatment, J. Membr. Sci. 479 (2015) 256-275.

[4] P. Li, K. Sun, J. Ouyang. Stretchable and Conductive Polymer Films Prepared by Solution Blending, ACS Appl. Mater. Interfaces (2015).

[5] G. Ren, Y. Miao, L. Qiao, Y. Qin, X. Wang, F. Wang. Toughening of amorphous poly(propylene carbonate) by rubbery CO2-based polyurethane: transition from brittle to ductile, RSC Adv. 5 (2015) 49979-49986.

[6] V. Kanyanta, A. Ivankovic. Mechanical characterisation of polyurethane elastomer for biomedical applications, J. Mech. Behav. Biomed Mater. 3 (2010) 51-62.

[7] N.G. McCrum, C.P. Buckley, C.B. Bucknall. Principles of Polymer Engineering. $2^{\text {nd }}$ ed., OUP Inc., NY, Oxford, 1997.

[8] S. Sen, S. Patil, D.S. Argyropoulos. Thermal properties of lignin in copolymers, blends, and composites: a review, Green Chem. (2015).

[9] P.-Y. Le Gac, M. Arhant, P. Davies, A. Muhr. Fatigue behavior of natural rubber in marine environment: Comparison between air and sea water, Mater. Design 65 (2015) 462-467.

[10] R.A. Ghunem, S.H. Jayaram, E.A. Cherney. The DC Inclined-Plane Tracking and Erosion Test and the Role of Inorganic Fillers in Silicone Rubber for DC Insulation, IEEE Elect. Insul. Mag. 31 (2015) 12-21.

[11] F. Tornuk, M. Hancer, O. Sagdic, H. Yetim. LLDPE based food packaging incorporated with nanoclays grafted with bioactive compounds to extend shelf life of some meat products, LWT-Food Sci. Technol. 64 (2015) 540-546.

[12] M. Gorji, A.A.A. Jeddi, A.A. Gharehaghaji. Fabrication and characterization of polyurethane electrospun nanofiber membranes for protective clothing applications, J. Appl. Polym. Sci. 125 (2012) 4135-4141.

[13] H. Golbakshi, M. Namjoo. Investigating tempearture Effects on Mechanical Behavior of Rubber Compounds Embedded in Composite Structure of Pneumatic Tires, Int. J. Adv. Manuf. Technol. 7 (2014) 19-25.

[14] H. Liu, X. Wang, D. Wu. Synthesis of a novel linear polyphosphazene-based epoxy resin and its application in halogen-free flame-resistant thermosetting systems, Polym. Degrad. Stabil. 118 (2015) 4558.

[15] M. Akiba, A.S. Hashim. Vulcanization and crosslinking in elastomers, Prog. Polym. Sci. 22 (1997) 475521.

[16] L. Tan, J. Hu, H. Zhao. Design of bilayered nanofibrous mats for wound dressing using an electrospinning technique, Mater. Lett. 156 (2015) 46-49.

[17] O.M. Yaghi, G.M. Li, H.L. Li. Selective Binding and Removal of Guests in a Microporous MetalOrganic Framework, Nature 378 (1995) 703-706.

[18] J.C. Tan, A.K. Cheetham. Mechanical properties of hybrid inorganic-organic framework materials: establishing fundamental structure-property relationships, Chem. Soc. Rev. 40 (2011) 1059-1080.

[19] D.-B.D. Li-Li Wen, Chun-Ying Dian, Yi-Zhi Li, Zheng-Fang Tian, and Qing-Jin Meng. 1D Helix, 2D Brick-Wall and Herringbone, and 3D Interpenetration $\mathrm{d}^{10}$ Metal Organic Framework Structures Assembled from Pyriddine-2,6-dicarboxylic Acid N-Oxide, Inorg. Chem. 44 (2005) 10.

[20] K.S. Park, Z. Ni, A.P. Cote, J.Y. Choi, R. Huang, F.J. Uribe-Romo, H.K. Chae, M. O'Keeffe, O.M. Yaghi. Exceptional chemical and thermal stability of zeolitic imidazolate frameworks, Proc. Natl. Acad. Sci. USA 103 (2006) 10186-10191. 
[21] O.K. Farha, J.T. Hupp. Rational design, synthesis, purification, and activation of metal-organic framework materials, Accounts Chem. Res. 43 (2010) 1166-1175.

[22] Y.J. Colon, R.Q. Snurr. High-throughput computational screening of metal-organic frameworks, Chem. Soc. Rev. 43 (2014) 5735-5749.

[23] R. Banerjee, A. Phan, B. Wang, C. Knobler, H. Furukawa, M. O'Keeffe, O.M. Yaghi. High-throughput synthesis of zeolitic imidazolate frameworks and application to CO2 capture, Science 319 (2008) 939943.

[24] S.S.-Y. Chui, S.M.-F. Lo, J.P.H. Charmant, A.G. Orpen, I.D. Williams. A Chemically Functionalizable Nanoporous Material $\left[\mathrm{Cu}_{3}(\mathrm{TMA})_{2}(\mathrm{H} 2 \mathrm{O})_{3}\right]_{n}$, Science 283 (1999) 1148-1150.

[25] J.H. Cavka, S. Jakobsen, U. Olsbye, N. Guillou, C. Lamberti, S. Bordiga, K.P. Lillerud. A new zirconium inorganic building brick forming metal organic frameworks with exceptional stability, J. Am. Chem. Soc. 130 (2008) 13850-13851.

[26] H.B. Tanh Jeazet, C. Staudt, C. Janiak. Metal-organic frameworks in mixed-matrix membranes for gas separation, Dalton Trans. 41 (2012) 14003-14027.

[27] L.J. Murray, M. Dinca, J.R. Long. Hydrogen storage in metal-organic frameworks, Chem. Soc. Rev. 38 (2009) 1294-1314.

[28] F. Cacho-Bailo, G. Caro, M. Etxeberria-Benavides, O. Karvan, C. Tellez, J. Coronas. High selectivity ZIF-93 hollow fiber membranes for gas separation, Chem. Commun. 51 (2015) 11283-11285.

[29] W. Xia, A. Mahmood, R. Zou, Q. Xu. Metal-organic frameworks and their derived nanostructures for electrochemical energy storage and conversion, Energy Environ. Sci. 8 (2015) 1837-1866.

[30] J.C. Tan, B. Civalleri. Metal-Organic Frameworks and Hybrid Materials: From Fundamentals and Applications, CrystEngComm 17 (2015) 197-198.

[31] M.D. Allendorf, V. Stavila. Crystal engineering, structure-function relationships, and the future of metal-organic frameworks, CrystEngComm 17 (2015) 229-246.

[32] P. Falcaro, R. Ricco, A. Yazdi, I. Imaz, S. Furukawa, D. Maspoch, R. Ameloot, J.D. Evans, C.J. Doonan. Application of metal and metal oxide nanoparticles@ MOFs, Coord. Chem. Rev. 307 (2016) 237-254.

[33] N. Stock, S. Biswas. Synthesis of metal-organic frameworks (MOFs): routes to various MOF topologies, morphologies, and composites, Chem. Rev. 112 (2012) 933-969.

[34] C. Xin, H. Zhan, X. Huang, H. Li, N. Zhao, F. Xiao, W. Wei, Y. Sun. Effect of various alkaline agents on the size and morphology of nano-sized HKUST-1 for CO2adsorption, RSC Adv. 5 (2015) 2790127911.

[35] A.K. Chaudhari, I. Han, J.C. Tan. Multifunctional Supramolecular Hybrid Materials Constructed from Hierarchical Self-Ordering of In Situ Generated Metal-Organic Framework (MOF) Nanoparticles, Adv. Mater. 27 (2015) 4438-4446.

[36] S. Liu, L. Sun, F. Xu, J. Zhang, C. Jiao, F. Li, Z. Li, S. Wang, Z. Wang, X. Jiang, H. Zhou, L. Yang, C. Schick. Nanosized $\mathrm{Cu}-\mathrm{MOF}$ induced by graphene oxide and enhanced gas storage capacity, Energy Environ. Sci. 6 (2013) 818.

[37] N.P. Panapitiya, S.N. Wijenayake, Y. Huang, D. Bushdiecker, D. Nguyen, C. Ratanawanate, G.J. Kalaw, C.J. Gilpin, I.H. Musselman, K.J. Balkus, J.P. Ferraris. Stabilization of immiscible polymer blends using structure directing metal organic frameworks (MOFs), Polymer 55 (2014) 2028-2034.

[38] R.C. Huxford, J. Della Rocca, W. Lin. Metal-organic frameworks as potential drug carriers, Curr. Opin. Chem. Biol. 14 (2010) 262-268.

[39] R.M. Jones. Mechanics of Composites Materials. $2^{\text {nd }}$ ed., Taylor \& Francis, 1999.

[40] L.H. Sperling. Introduction to Physical Polymer Science. $4^{\text {th }}$ ed., Wiley-Interscience, 2006.

[41] H.C. Zhou, J.R. Long, O.M. Yaghi. Introduction to metal-organic frameworks, Chem. Rev. 112 (2012) 673-674.

[42] S. Shahid, K. Nijmeijer. Performance and plasticization behavior of polymer-MOF membranes for gas separation at elevated pressures, J. Membr. Sci. 470 (2014) 166-177.

[43] X.L. Yang, C. Zou, Y. He, M. Zhao, B. Chen, S. Xiang, M. O'Keeffe, C.D. Wu. A stable microporous mixed-metal metal-organic framework with highly active $\mathrm{Cu} 2+$ sites for efficient cross-dehydrogenative coupling reactions, Chem. Eur. J. 20 (2014) 1447-1452.

[44] R. Grunker, V. Bon, P. Muller, U. Stoeck, S. Krause, U. Mueller, I. Senkovska, S. Kaskel. A new metalorganic framework with ultra-high surface area, Chem. Commun. (Camb.) 50 (2014) 3450-3452.

[45] B. Barth, M. Mendt, A. Pöppl, M. Hartmann. Adsorption of nitric oxide in metal-organic frameworks: Low temperature IR and EPR spectroscopic evaluation of the role of open metal sites, Microporous Mesoporous Mat. 216 (2015) 97-110.

[46] M. Hegde, S. Shahid, B. Norder, T.J. Dingemans, K. Nijmeijer. Gas transport in metal organic framework-polyetherimide mixed matrix membranes: The role of the polyetherimide backbone structure, Polymer 81 (2015) 87-98. 
[47] X. Liang, F. Zhang, W. Feng, X. Zou, C. Zhao, H. Na, C. Liu, F. Sun, G. Zhu. From metal-organic framework (MOF) to MOF-polymer composite membrane: enhancement of low-humidity proton conductivity, Chem. Sci. 4 (2013) 983-992.

[48] Q.L. Zhu, Q. Xu. Metal-organic framework composites, Chem. Soc. Rev. 43 (2014) 5468-5512.

[49] M.R. Ryder, B. Civalleri, T.D. Bennett, S. Henke, S. Rudic, G. Cinque, F. Fernandez-Alonso, J.C. Tan. Identifying the role of terahertz vibrations in metal-organic frameworks: from gate-opening phenomenon to shear-driven structural destabilization, Phys. Rev. Lett. 113 (2014) 215502.

[50] E.M. Mahdi, J.-C. Tan. Mixed-matrix membranes of zeolitic imidazolate framework (ZIF-8)/Matrimid nanocomposite: Thermo-mechanical stability and viscoelasticity underpinning membrane separation performance, J. Membr. Sci. 498 (2016) 276-290.

[51] M.S. Denny, Jr., S.M. Cohen. In Situ Modification of Metal-Organic Frameworks in Mixed-Matrix Membranes, Angew. Chem. Int. Ed. Engl. 54 (2015) 9029-9032.

[52] M.J.C. Ordoñez, K.J. Balkus, J.P. Ferraris, I.H. Musselman. Molecular sieving realized with ZIF8/Matrimid® mixed-matrix membranes, J. Membr. Sci. 361 (2010) 28-37.

[53] A. Kathuria, M.G. Abiad, R. Auras. Toughening of poly(l-lactic acid) with Cu3BTC2 metal organic framework crystals, Polymer 54 (2013) 6979-6986.

[54] M. Fang, C. Wu, Z. Yang, T. Wang, Y. Xia, J. Li. ZIF-8/PDMS mixed matrix membranes for propane/nitrogen mixture separation: Experimental result and permeation model validation, J. Membr. Sci. 474 (2015) 103-113.

[55] H. Zhu, Q. Zhang, S. Zhu. Preparation of raspberry-like ZIF-8/PS composite spheres via dispersion polymerization, Dalton Trans. (2015).

[56] J. Cravillon, S. Münzer, S.-J. Lohmeier, A. Feldhoff, K. Huber, M. Wiebcke. Rapid Room-Temperature Synthesis and Characterization of Nanocrystals of a Prototypical Zeolitic Imidazolate Framework, Chem. Mater. 21 (2009) 1410-1412.

[57] Q. Song, S.K. Nataraj, M.V. Roussenova, J.C. Tan, D.J. Hughes, W. Li, P. Bourgoin, M.A. Alam, A.K. Cheetham, S.A. Al-Muhtaseb, E. Sivaniah. Zeolitic imidazolate framework (ZIF-8) based polymer nanocomposite membranes for gas separation, Energy Environ. Sci. 5 (2012) 8359.

[58] R.D. Widdle, A.K. Bajaj, P. Davies. Measurement of the Poisson's ratio of flexible polyurethane foam and its influence on a uniaxial compression model, Int. J. Eng. Sci. 46 (2008) 31-49.

[59] R.W. Ogden. Large deformation isotropic elasticity - on the correlation of theory and experiment for incompressible rubberlike solids, Proc. R. Soc. Lond. A. 326 (1972) 19.

[60] T. Yang, G.M. Shi, T.-S. Chung. Symmetric and Asymmetric Zeolitic Imidazolate Frameworks (ZIFs)/Polybenzimidazole (PBI) Nanocomposite Membranes for Hydrogen Purification at High Temperatures, Adv. Energy Mater. 2 (2012) 1358-1367.

[61] A.F. Bushell, M.P. Attfield, C.R. Mason, P.M. Budd, Y. Yampolskii, L. Starannikova, A. Rebrov, F. Bazzarelli, P. Bernardo, J. Carolus Jansen, M. Lanč, K. Friess, V. Shantarovich, V. Gustov, V. Isaeva. Gas permeation parameters of mixed matrix membranes based on the polymer of intrinsic microporosity PIM-1 and the zeolitic imidazolate framework ZIF-8, J. Membr. Sci. 427 (2013) 48-62.

[62] Y. Pan, D. Heryadi, F. Zhou, L. Zhao, G. Lestari, H. Su, Z. Lai. Tuning the crystal morphology and size of zeolitic imidazolate framework-8 in aqueous solution by surfactants, CrystEngComm 13 (2011) 6937.

[63] S. Hwang, W.S. Chi, S.J. Lee, S.H. Im, J.H. Kim, J. Kim. Hollow ZIF-8 nanoparticles improve the permeability of mixed matrix membranes for CO2/CH4 gas separation, J. Membr. Sci. 480 (2015) 11-19.

[64] S.J.K. Jensen, T.H. Tang, I.G. Csizmadia. Hydrogen-bonding ability of a methyl group, J. Phys. Chem. A 107 (2003) 8975-8979.

[65] D. Ballestero, R. Juan, A. Ibarra, C. Gómez-Giménez, C. Ruiz, B. Rubio, M. Teresa Izquierdo. Effect of thermal treatments on the morphology, chemical state and lattice structure of gold nanoparticles deposited onto carbon structured monoliths, Colloid. Surf. A 468 (2015) 140-150.

[66] R. Shankar, U. Sahoo, V. Shahi. Synthesis and Characterization of Fluorescent Polymer-Metal Nanocomposites Comprising Poly(silylene-co-silyne)s and Silver Nanoparticles, Macromolecules 44 (2011) 3240-3249.

[67] A.V. Tobolsky, D.W. Carlson, N. Indictor. Rubber Elasticity and Chain Configuration, J. Polym. Sci. 54 (1961) 18 .

[68] G.R. Deen, E.K. Lim, C.H. Mah, K.M. Heng. New Cationic Linear Copolymers and Hydrogels ofNVinyl Caprolactam andN-Acryloyl-N' -ethyl Piperazine: Synthesis, Reactivity, Influence of External Stimuli on the LCST and Swelling Properties, Ind. Eng. Chem. Res. 51 (2012) 13354-13365.

[69] J.R. Collier. Polymer Deformation and Orientation, Ind. Eng. Chem. 61 (1969) 14.

[70] A. Kudasheva, S. Sorribas, B. Zornoza, C. Téllez, J. Coronas. Pervaporation of water/ethanol mixtures through polyimide based mixed matrix membranes containing ZIF-8, ordered mesoporous silica and ZIF8-silica core-shell spheres, J. Chem. Technol. Biot. 90 (2015) 669-677. 
[71] E.V. Perez, K.J. Balkus, J.P. Ferraris, I.H. Musselman. Mixed-matrix membranes containing MOF-5 for gas separations, J. Membr. Sci. 328 (2009) 165-173.

[72] J.C. Tan, B. Civalleri, C.C. Lin, L. Valenzano, R. Galvelis, P.F. Chen, T.D. Bennett, C. MellotDraznieks, C.M. Zicovich-Wilson, A.K. Cheetham. Exceptionally low shear modulus in a prototypical imidazole-based metal-organic framework, Phys. Rev. Lett. 108 (2012) 095502.

[73] J.C. Tan, T.D. Bennett, A.K. Cheetham. Chemical structure, network topology, and porosity effects on the mechanical properties of Zeolitic Imidazolate Frameworks, Proc. Natl. Acad. Sci. USA 107 (2010) 9938-9943.

[74] D. Hull, T.W. Clyne. An Introduction to Composite Materials. $2^{\text {nd }}$ ed., 1996.

[75] M.K. Sami Hietala, Eivind Skou, Matti Elomaa, and Franciska Sundholm. Thermal Stability of Styrene Grated and Sulfonated Proton Conducting Membranes based on poly(vinylidene flouride), J. Mater. Chem. 8 (1998) 6.

[76] S.J. Smith, B.P. Ladewig, A.J. Hill, C.H. Lau, M.R. Hill. Post-synthetic Ti exchanged UiO-66 metalorganic frameworks that deliver exceptional gas permeability in mixed matrix membranes, Sci. Rep. 5 (2015) 7823.

[77] S. Belhaj Messaoud, A. Takagaki, T. Sugawara, R. Kikuchi, S.T. Oyama. Mixed matrix membranes using SAPO-34/polyetherimide for carbon dioxide/methane separation, Sep. Purif. Technol. 148 (2015) 38-48.

[78] A.W.C.a.J.P. Redfern. Thermogravimetric analysis. A review, Analyst 88 (1963) 19.

[79] G. Trovati, E.A. Sanches, S.C. Neto, Y.P. Mascarenhas, G.O. Chierice. Characterization of polyurethane resins by FTIR, TGA, and XRD, J. Appl. Polym. Sci. 115 (2010) 263-268.

[80] C. Zhang, Y. Dai, J.R. Johnson, O. Karvan, W.J. Koros. High performance ZIF-8/6FDA-DAM mixed matrix membrane for propylene/propane separations, J. Membr. Sci. 389 (2012) 34-42.

[81] A.J. Crosby, J.Y. Lee. Polymer Nanocomposites: The "Nano" Effect on Mechanical Properties, Polym. Rev. 47 (2007) 217-229.

[82] R. Lakes. Viscoelastic Materials, Cambridge University Press, United States of America, 2009.

[83] D.G. Fesko, N.W. Tschoegl. Time-Temperature Superposition in Thermorheologically Complex Materials, J. Polym. Sci. Polym. Symp. 35 (1971) 51-\&.

[84] F. Schwarzl, A.J. Staverman. Time-Temperature Dependence of Linear Viscoelastic Behavior, J. Appl. Phys. 23 (1952) 838.

[85] C. Komalan, K.E. George, P.A.S. Kumar, K.T. Varughese, S. Thomas. Dynamic mechanical analysis of binary and ternary polymer blends based on nylon copolymer/EPDM rubber and EPM grafted maleic anhydride compatibilizer, Express Polym. Lett. 1 (2007) 641-653.

[86] F.G. Ornaghi, V. Pistor, R.V.B. de Oliveira. Thermoplastic polyurethane synthesis with modified montmorillonite prepared by torque rheometry: Investigation of morphological, thermal, chemical, and physical properties, J. Appl. Polym. Sci. 132 (2015) 42640-42650.

[87] X.Y. Chen, H. Vinh-Thang, D. Rodrigue, S. Kaliaguine. Amine-Functionalized MIL-53 Metal-Organic Framework in Polyimide Mixed Matrix Membranes for CO2/CH4Separation, Ind. Eng. Chem. Res. 51 (2012) 6895-6906.

[88] R.W. Baker. Future directions of membrane gas separation technology, Ind. Eng. Chem. Res. 41 (2002) 1393-1411.

[89] V. Abetz, T. Brinkmann, M. Dijkstra, K. Ebert, D. Fritsch, K. Ohlrogge, D. Paul, K.V. Peinemann, S. Pereira-Nunes, N. Scharnagl, M. Schossig. Developments in Membrane Research: from Material via Process Design to Industrial Application, Adv. Eng. Mater. 8 (2006) 328-358.

[90] B. Zornoza, C. Tellez, J. Coronas, J. Gascon, F. Kapteijn. Metal organic framework based mixed matrix membranes: An increasingly important field of research with a large application potential, Microporous Mesoporous Mat. 166 (2013) 67-78.

[91] E.A. Feijani, H. Mahdavi, A. Tavasoli. Poly(vinylidene fluoride) based mixed matrix membranes comprising metal organic frameworks for gas separation applications, Chem. Eng. Res. Des. 96 (2015) 87-102.

[92] N.B. McKeown, P.M. Budd. Polymers of intrinsic microporosity (PIMs): organic materials for membrane separations, heterogeneous catalysis and hydrogen storage, Chem. Soc. Rev. 35 (2006) 675683.

[93] J.X. Ren, A.S. Silva, R. Krishnamoorti. Linear viscoelasticity of disordered polystyrene-polyisoprene block copolymer based layered-silicate nanocomposites, Macromolecules 33 (2000) 3739-3746.

[94] D. Hua, Y.K. Ong, Y. Wang, T. Yang, T.-S. Chung. ZIF-90/P84 mixed matrix membranes for pervaporation dehydration of isopropanol, J. Membr. Sci. 453 (2014) 155-167. 


\section{Highlights}

- $\quad$ Hyperelastic polyurethane (PU)/ZIF-8 mixed-matrix nanocomposite membranes

- Determined viscoelasticity and thermo-mechanical responses up to $40 \mathrm{wt} . \%$

- Thermal stability enhancement and stabilisation of phases under dynamic stress

- Ductility of PU matrix confers mechanical resilience and membrane toughness

- Secondary molecular interactions control thermo-mechanical performance 